\title{
Advanced MIMO Techniques: Polarization Diversity and Antenna Selection
}

\author{
Kosai RAOOF${ }^{1}$, Maha BEN ZID ${ }^{2,4}$, Nuttapol PRAYONGPUN ${ }^{3}$ and Ammar \\ BOUALLEGUE ${ }^{4}$ \\ 1,2 UJF-Grenoble I, Gipsa Lab - UMR 5216 CNRS \\ ${ }^{3}$ College of Industrial Technology \\ ${ }^{4}$ National Engineering School of Tunis (ENIT), 6'Com Lab \\ ${ }^{1,2}$ France \\ ${ }^{3}$ Thailand \\ ${ }^{4}$ Tunisia
}

\section{Introduction}

This chapter is attempted to provide a survey of the advanced concepts and related issues involved in Multiple Input Multiple Output (MIMO) systems. MIMO system technology has been considered as a really significant foundation on which to build the next and future generations of wireless networks. The chapter addresses advanced MIMO techniques such as polarization diversity and antenna selection. We gradually provide an overview of the MIMO features from basic to more advanced topics. The first sections of this chapter start by introducing the key aspects of the MIMO theory. The MIMO system model is first presented in a generic way. Then, we proceed to describe diversity schemes used in MIMO systems. MIMO technology could exploit several diversity techniques beyond the spatial diversity. These techniques essentially cover frequency diversity, time diversity and polarization diversity. We further provide the reader with a geometrically based models for MIMO systems. The virtue of this channel modeling is to adopt realistic methods for modeling the spatio-temporal channel statistics from a physical wave-propagation viewpoint. Two classes for MIMO channel modeling will be described. These models involve the Geometry-based Stochastic Channel Models (GSCM) and the Stochastic channel models. Besides the listed MIMO channel models already described, we derive and discuss capacity formulas for transmission over MIMO systems. The achieved MIMO capacities highlight the potential of spatial diversity for improving the spectral efficiency of MIMO channels. When Channel State Information (CSI) is available at both ends of the transmission link, the MIMO system capacity is optimally derived by using adaptive power allocation based on water-filling technique. The chapter continues by examining the combining techniques for multiple antenna systems. Combining techniques are motivated for MIMO systems since they enable the signal to noise ratio (SNR) maximization at the combiner output. The fundamental combing techniques are the Maximal Ratio Combining (MRC), the Selection Combining (SC) and the Equal Gain Combining(EGC). Once the combining techniques are analyzed, the reader is introduced to the beamforming processing as an optimal strategy for combining. The use of multiple antennas significantly 
improves the channel spectral efficiency. Nevertheless, this induces higher system complexity of the communication system and the communication system performance is effected due to correlation between antennas that need to be deployed at the same terminal. As such, the antenna selection algorithm for MIMO systems is presented. To elaborate on this point, we introduce Space time coding techniques for MIMO systems and we evaluate by simulation the performance of the communication system. Next, we emphasis on multi polarization techniques for MIMO systems. As a background, we presume that the reader has a thorough understanding of antenna theory. We recall the basic antenna theory and concepts that are used throughout the rest of the chapter. We rigorously introduce the 3D channel model over the Non-Line of Sight (NLOS) propagation channel for MIMO system with polarized antennas. We treat the depolarization phenomena and we study its effect on MIMO system capacity. The last section of the chapter provides a scenario for collaborative sensor nodes performing distributed MIMO system model which is devoted to sensor node localization in Wireless Sensor Networks. The localization algorithm is based on beamforming processing and was tested by simulation. Our chapter provides the reader by simulation examples for almost all the topics that have been treated for MIMO system development and key issues affecting achieved performance.

\section{MIMO literature and mathematical model}

This section gives an overview of the MIMO literature. MIMO technology has been a subject of research since the last decade of the twentieth century. In 1984, Jack Winters at Bell Laboratories wrote a patent on wireless communications using multiple antennas. Jack Winters in (Winters, 1987) presented a study of the fundamental limits on the data rate of multiple antenna systems in a Rayleigh fading environment. The concept of MIMO was introduced for two basic communication systems which are a communication system between multiple mobiles and a base station with multiple antennas and another one between two mobiles with multiple antennas. In 1993, Arogyaswami Paulraj and Thomas Kailath proposed the concept of spatial multiplexing using MIMO. They filed a patent on spatial multiplexing emphasized applications to wireless broadcast. Several articles which focused on MIMO concept were published in the period from 1986 to 1995. We mainly cite the article of Emre Teletar titled "Capacity of multi-antenna gaussian channels" (Telatar, 1995). This was followed by the work of Greg Raleigh and Gerard Joseph Foschini in 1996 (Foshini, 1996) which invented new approaches involving space time coding techniques. These approaches were proved to increase the spectral efficiency of MIMO systems (Raleigh \& John, 1998). In 1999, Thomas L. Marzetta and Bertrand M. Hochwald published an article (Marzetta \& Hochwald, 1999) which provides a rigorous study on the MIMO Rayleigh fading link taking into consideration information theory aspects. Afterwards, MIMO communication techniques have been developed and brought completely on new perspectives wireless channels. The first commercial MIMO system was developed in 2001 by Iospan Wireless Inc. Since 2006, several companies such as Broadcom and Intel have concerned a novel communication technique based on the MIMO technology for improving the performance of wireless Local Area Network(LAN) systems. The new standard of wireless LAN systems is named IEEE 802.11n. MIMO technology has attracted more attention in wireless communications. In fact, it was used to boost the link capacity and to enhance the reliability of the communication link. MIMO scheme is the major candidate technology in various standard proposals for the fourth-generation of wireless communication systems. Enhanced techniques for MIMO communications led to advanced technologies for achieving successful radio transmission. It 
promises significant improvements in spectral efficiency and network coverage. We mainly cite multiple access MIMO systems, Ad-hoc MIMO , cooperative MIMO (Wang et al., 2010) and cooperative MIMO in sensor networks (Shuguang et al., 2004). Note that cooperative MIMO systems use multiple distributed transmitting devices to improve Quality of Service (QoS) at one/multiple receivers. This was shown to bring saves in energy and to improve the link reliability in Wireless Sensor Network (WSN) where multiple sensor nodes can be cooperatively functioned. In the following, we introduce the mathematical model for MIMO systems. We briefly describe the flat fading MIMO channel and the continuous time delay MIMO channel model.

\section{Flat fading MIMO channel}

Transmit antennas Receive antennas

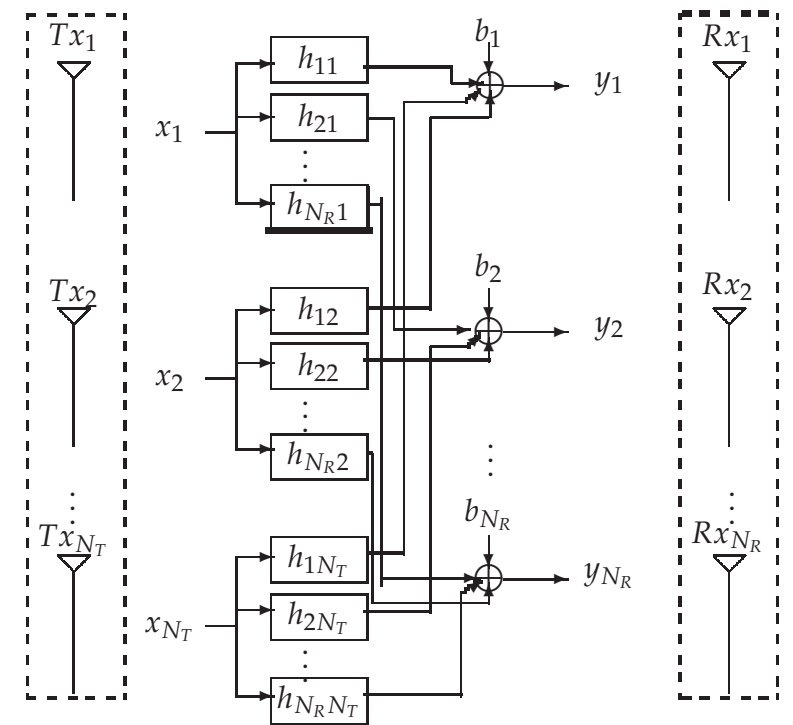

Fig. 1. Generic MIMO system model

Generic MIMO system with $N_{T}$ transmit antennas and $N_{R}$ receive antennas is depicted in Fig. 1. Such model is typically used for cases where the frequency domain channel transfer function remains approximately constant over the bandwidth of the transmitted waveform and is referred to as the flat fading scenario. The input output relationship for this MIMO system is defined as :

$$
\mathbf{y}=\mathbf{H x}+\mathbf{b}
$$

where :

- $\mathbf{H}$ is the $\left(N_{R} \times N_{T}\right)$ complex channel matrix described as :

$$
\mathbf{H}=\left[\mathbf{h}_{1}, \ldots, \mathbf{h}_{N_{T}}\right]
$$

$\mathbf{h}_{p}=\left[h_{1 p}, \ldots, h_{N_{R} p}\right]^{T} ; \quad p=1, \ldots, N_{T}$ is the complex channel vector which links the transmit antenna $T x_{p}$ to the $N_{R}$ receive antennas $R x_{1}, \ldots, R x_{N_{R}}$. 
- $\mathbf{x}=\left[x_{1}, \ldots, x_{N_{T}}\right]^{T}$ is the complex vector for the transmitted signal

- $\mathbf{y}=\left[y_{1}, \ldots, y_{N_{R}}\right]^{T}$ is the complex vector for the received signal

- $\mathbf{b}=\left[b_{1}, \ldots, b_{N_{R}}\right]^{T}$ is the complex vector for the additive noise signal

At the receive antenna $R x_{q}$, the received signal is expressed as :

$$
y_{q}=\sum_{p=1}^{N_{T}} h_{q p} x_{p}+b_{q} \quad ; q=1, \ldots, N_{R}
$$

In the literature, other cases of simplified MIMO systems are also explained :

- Single Input Multiple Output (SIMO) is a simplified form of MIMO systems where the transmitter system has a single antenna.

- Multiple Input Single Output (MISO) is a form of MIMO systems where the receiver system has a single antenna.

- When neither the receiver nor the transmitter has multiple antennas, the radio system is called Single Input Single Output (SISO) system.

The listed multiple antenna models are represented in Fig. 2.

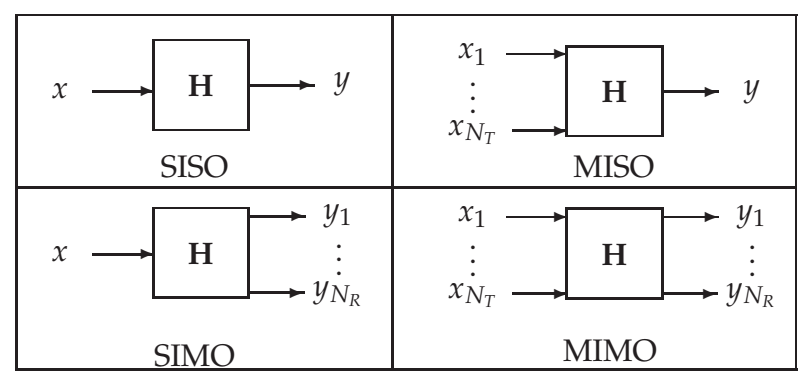

Fig. 2. Multiple antenna system

\section{Continuous time delay MIMO channel model}

The continuous time delay MIMO channel model describes the dynamic behavior of the MIMO channel. The spatio-temporel signal output $\mathbf{y}(t)$ is expressed in terms of the spatio-temporel signal input $\mathbf{x}(t)$, the $\left(N_{R} \times N_{T}\right)$ MIMO channel $\mathbf{H}$ associated time delay and the noise signal $\mathbf{b}(t)$ as :

$$
\mathbf{y}(t)=\int_{\tau} \mathbf{H}(t, \tau) \mathbf{x}(t-\tau) d \tau+\mathbf{b}(t)
$$

$\tau$ is the time delay.

\section{Diversity schemes}

This section is intended to present methods for improving the reliability of communication system by using different types of diversity. 


\subsection{Spatial diversity}

The use of multiple antennas in MIMO systems improves the performance of communication systems. Signal will not suffer the same level of attenuation as it propagates along different paths. The use of multiple antennas is called spatial diversity. Joint transmit and receive diversity are carried out in MIMO systems. Nevertheless, the spatial diversity scheme can be efficiently exploited when the antenna array configuration at receive and transmit sides is properly performed to the propagation environment characteristic. This could be achieved if multiple branches which are combined are ideally uncorrelated in order to reduce probability for deep fades in fading channels.

\section{Diversity gain}

The spatial diversity systems are known for their reliability through the use of multiple receive and transmit antenna arrays. The system reliability is represented by the diversity gain. Diversity gain measures the increase of the error rate against the SNR and could be expressed as the slope of the error rate as a function of SNR when SNR tends to infinity. A tractable definition of the diversity gain is (Jafarkhani, 2005):

$$
d=-\lim _{\mathrm{SNR} \rightarrow \infty} \frac{\log \left(P_{e}(\mathrm{SNR})\right)}{\log (\mathrm{SNR})}
$$

$P_{e}(\mathrm{SNR})$ denotes the error rate measured at a fixed SNR value. A MIMO system with $N_{T}$ transmit antennas and $N_{R}$ receive antennas can achieve a maximum diversity gain of $N_{T} \times$ $N_{R}$.

\section{Multiplexing gain}

Thanks to the use of multiple antennas, MIMO systems perform spatial multiplexing. Independent and separately data signals called streams are transmitted from each transmit antenna. The data streams arrived at the receiver are demultiplexed and the maximum number of independent transmission channels or degrees of freedom $\operatorname{are} \min \left(N_{R}, N_{T}\right)$ (Zheng $\&$ Tse, 2003). Such technique leads to an increase in the system spectral efficiency without any need neither for additional bandwidth nor for additional power allocation. The spatial multiplexing order is expressed as :

$$
r=\lim _{\mathrm{SNR} \rightarrow \infty} \frac{\mathrm{R}(\mathrm{SNR})}{\log (\mathrm{SNR})}
$$

$\mathrm{R}(\mathrm{SNR})$ denotes the capacity for a given SNR value.

\section{Diversity-Multiplexing trade-off}

We should note that there is a compromise between maximizing the diversity gain so that to increase the link reliability against fading and maximizing the multiplexing gain in order to achieve the best spectral efficiency. This trade-off is expressed as :

$$
d(r)=\left(N_{T}-r\right)\left(N_{R}-r\right) \quad ; r=0, \ldots, \min \left(N_{R}, N_{T}\right)
$$

This implies that if $r$ pairs of antennas (Each pair consists of one transmit antenna and one receive antenna) are exploited for spatial multiplexing, it remains $\left(N_{T}-r\right)$ transmit antennas and $\left(N_{R}-r\right)$ receive antennas to be exploited for diversity gain. Nevertheless, coding techniques could be used as a solution for inherent diversity-multiplexing trade-off (Freitas et al., 2005). 


\subsection{Temporal diversity and Space Time processing for MIMO systems}

If channel varies in time, repeated signal versions can benefit from temporal diversity if they are sent at different time intervals that is higher than the time coherence of the channel.

\section{Space Time processing for MIMO systems}

MIMO system can still achieve both spatial diversity and temporal diversity by exploiting Space Time (ST) coding (Fig. 3). Let us review the flat fading model. The complex channel matrix $\mathbf{H}\left(N_{R} \times N_{T}\right)$ is expressed as :

$$
\mathbf{H}=\left(\begin{array}{cccc}
h_{11} & h_{12} & \ldots & h_{1 N_{T}} \\
h_{21} & h_{22} & \ldots & h_{2 N_{T}} \\
\vdots & \vdots & \ddots & \vdots \\
h_{N_{R} 1} & h_{N_{R}} & \ldots & h_{N_{R} N_{T}}
\end{array}\right)
$$

Given a block time of length $\mathrm{L}$, at time $t$, the transmitted signal is expressed as :

$$
\mathbf{x}^{(t)}=\left[x_{1}^{(t)} \ldots, x_{N_{T}}^{(t)}\right]^{T} ; t=1, \ldots, \mathrm{L}
$$

The input array signal $\mathbf{X}\left(N_{T} \times L\right)$ is given by :

$$
\mathbf{X}=\left(\begin{array}{cccc}
x_{1}^{(1)} & x_{1}^{(2)} & \ldots & x_{1}^{(L)} \\
x_{2}^{(1)} & x_{2}^{(2)} & \ldots & x_{2}^{(L)} \\
\vdots & \vdots & \ddots & \vdots \\
x_{N_{T}}^{(1)} & x_{N_{T}}^{(2)} & \ldots & x_{N_{T}}^{(L)}
\end{array}\right)
$$

The received signal matrix $\mathbf{Y}\left(N_{R} \times L\right)$ is expressed as :

$$
\mathbf{Y}=\left(\begin{array}{cccc}
y_{1}^{(1)} & y_{1}^{(2)} & \ldots & y_{1}^{(\mathrm{L})} \\
y_{2}^{(1)} & y_{2}^{(2)} & \ldots & y_{2}^{(\mathrm{L})} \\
\vdots & \vdots & \ddots & \vdots \\
y_{N_{R}}^{(1)} & y_{N_{R}}^{(2)} & \ldots & y_{N_{R}}^{(\mathrm{L})}
\end{array}\right)
$$

The noise signal matrix $\mathbf{B}\left(N_{R} \times L\right)$ is :

$$
\mathbf{B}=\left(\begin{array}{cccc}
b_{1}^{(1)} & b_{1}^{(2)} & \ldots & b_{1}^{(\mathrm{L})} \\
b_{2}^{(1)} & b_{2}^{(2)} & \ldots & b_{2}^{(\mathrm{L})} \\
\vdots & \vdots & \ddots & \vdots \\
b_{N_{R}}^{(1)} & b_{N_{R}}^{(2)} & \ldots & b_{N_{R}}^{(\mathrm{L})}
\end{array}\right)
$$

The input output relationship of such system is given by :

$$
\mathbf{Y}=\mathbf{H} \cdot \mathbf{X}+\mathbf{B}
$$

Thereafter, the received signal at time $t$ at the receiving antenna $R x_{q}$ is expressed as :

$$
y_{q}^{(t)}=\sum_{p=1}^{N_{T}} h_{q p} x_{p}^{(t)}+b_{q}^{(t)} ; \quad t=1, \ldots, L ; \quad q=1, \ldots, N_{R}
$$


Thus, ST coding is a class of a linear processing design. The transmitted matrix $\mathbf{X}$ is referred as the ST code. ST codes are designed in order to achieve both maximum coding gain and diversity gain. Thereafter, two main criteria should be satisfied when ST coding is performed. These criteria are referred as the Rank criterion and the Determinant criterion (Tarokh et al., 1998). Recently many types of ST coding structures were invented. Nevertheless, ST codes

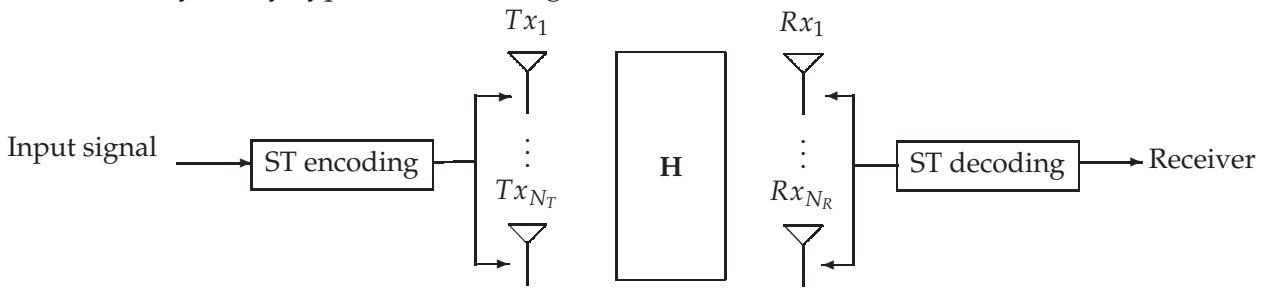

Fig. 3. ST coding for MIMO systems

may be split into two main types which are listed in the following :

1. Space Time Trellis Code (STTC) was invented by Vahid Tarokh in 1998. This coding scheme transmits multiple redundant copies of trellis code which are distributed in time and space. Vahid Tarokh gave a detailed description for trellis code construction in (Tarokh et al., 1998)

2. Space Time Block Code (STBC) aims to provide a diversity gain by transmitting block codes distributed over the transmit antennas. Development of STBC is based on complex orthogonal design. STBC for communication over Rayleigh fading channels was introduced in (Tarokh et al., 1999). The most famous orthogonal STBC (OSTBC) design is the Alamouti scheme which was invented in 1998 for a MIMO system when two-branch transmit diversity with one receive antenna and two-branch transmit diversity with two receive antennas are considered (Alamouti, 1998). At the receiver, the transmitted signal can be easily recovered due to the orthogonality of ST code. Thus, OSTBCs have received much attention from the coding community as compared to STTCs owing to their simple design and low complexity receivers (Ghrayeb, 2006). Performance analysis in terms of BER for various OSTBCs was derived in (Tran \& Sesay, 2003). It was shown that we can obtain gain from OSTBCs if appropriate number of transmit antennas are deployed.

For more detailed lecture about the listed ST codes, the reader could refer to (Tarokh et al., 1998) (Tarokh et al., 1999) and (Vucetic, B. \& Yuan, J., 2003). The simplest Alamouti scheme was presented in (Alamouti, 1998). For the case of two receive antennas, if we consider two symbols $x_{1}$ and $x_{2}$, then :

- At time slot $1, x_{1}$ and $x_{2}$ are transmitted simultaneously from $T x_{1}$ and $T x_{2}$.

- At time slot 2, $-x_{2}^{*}$ and $x_{1}^{*}$ are transmitted simultaneously from $T x_{1}$ and $T x_{2}$.

The input output relationship involving two receive antennas is expressed as :

$$
\left(\begin{array}{c}
y_{1}^{(1)} \\
y_{2}^{(1)} \\
\left(y_{1}^{(2)}\right)^{*} \\
\left(y_{2}^{(2)}\right)^{*}
\end{array}\right)=\left(\begin{array}{cc}
h_{11} & h_{12} \\
h_{21} & h_{22} \\
h_{12}^{*} & -h_{11}^{*} \\
h_{22}^{*} & -h_{21}^{*}
\end{array}\right) \cdot\left(\begin{array}{c}
x_{1} \\
x_{2}
\end{array}\right)+\left(\begin{array}{c}
b_{1}^{(1)} \\
b_{2}^{(1)} \\
\left(b_{1}^{(2)}\right)^{*} \\
\left(b 2^{(2)}\right)^{*}
\end{array}\right)
$$


Time

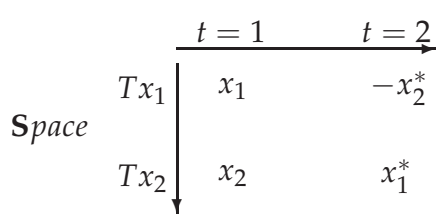

Fig. 4. Alamouti code

Let us denote :

$$
\text { Hequ }=\left(\begin{array}{cc}
h_{11} & h_{12} \\
h_{21} & h_{22} \\
h_{12}^{*} & -h_{11}^{*} \\
h_{22}^{*} & -h_{21}^{*}
\end{array}\right)
$$

The estimated transmitted signal at the receiver is given by :

$$
\left(\begin{array}{c}
\hat{x}_{1} \\
\hat{x}_{2}^{*}
\end{array}\right)=\mathbf{H}^{+}\left(\begin{array}{c}
y_{1}^{(1)} \\
y_{2}^{(1)} \\
\left(y_{1}^{(2)}\right)^{*} \\
\left(y_{2}^{(2)}\right)^{*}
\end{array}\right)
$$

where $\mathbf{H}^{+}$is the pseudo inverse matrix of Hequ. Simulation results of the Alamouti scheme for both MIMO systems $\mathrm{MIMO}(1 \times 2)$ and $\mathrm{MIMO}(2 \times 2)$ in case of fading Rayleigh channel with Additive White Gaussian Noise(AWGN) are depicted in Fig. 5. Transmitted data signals are Binary Phase Shift Keying (BPSK) modulated.

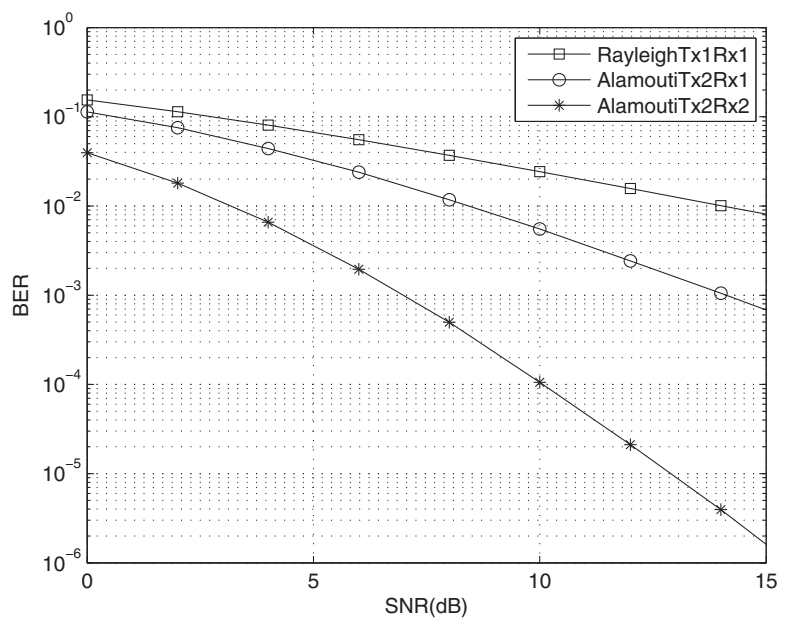

Fig. 5. BER curves for BPSK modulated signal with Alamouti coding 


\subsection{Frequency diversity}

Frequency diversity relies on the fact that signals are transmitted on different frequencies so that different multipath structures in the propagation media are exploited. Transmitting signals on different frequencies are referred as multicarrier transmission. One special case of multicarrier transmission is Orthogonal Frequency Division Multiplexing (OFDM). OFDM has got a great interest by the researchers and it was shown that using this type of modulation gives a significant performance increase in wireless communications. OFDM modulation technique was adopted by IEEE802.11a and IEEE 802.11b wireless LAN standards. When using the OFDM technique, a single data stream is transmitted over a number of lower rate carriers. This can be considered as a form of frequency multiplexing that could be efficient for wide band communication. The signal frequency band is divided into several frequency subchannels in order to get narrow band channels. Orthogonality between different modulated carriers is imposed in order to avoid overlapping subchannels. Therefore, signals are received without adjacent carrier interference. Both transmitter and receiver are implemented using respectively the Inverse Fast Fourier Transform (IFFT) and Fast Fourier Transform (FFT) techniques. The OFDM transmission scheme introduces guard bands / cyclic prefix between the different carriers. This lowers the spectrum efficiency but it eliminates the Inter Symbol Interference (ISI). It should be noted that when using OFDM technique, channel equalization becomes simpler. Simulation of the OFDM system was performed in order to measure the performance of such technique and compare it to the single carrier system. The simulated OFDM system is given by Fig. 6 .

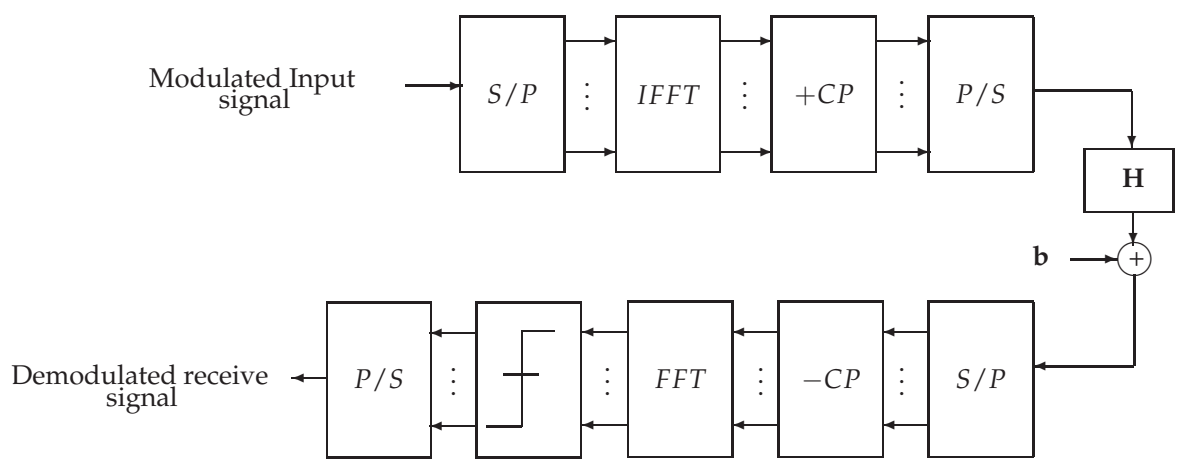

Fig. 6. General structure of OFDM system

- $P / S$ : Parallel to serial conversion

- $S$ / P: Serial to parallel conversion

- $+C P$ : Adding the cyclic prefix

- $-C P$ : Removing the cyclic prefix

- H: Channel matrix

- $\mathbf{b}$ : Additive noise

For simulation, we consider a BPSK modulation scheme, a FFT length equal to 52 and a FFT size of 64 . The simulation results are given by Fig. 7. OFDM can be used in conjunction with a MIMO system. MIMO-OFDM (See Fig. 8) is interesting for high data rate systems. A design and simulation of MIMO-OFDM was introduced in (Yu et al., 2004). 


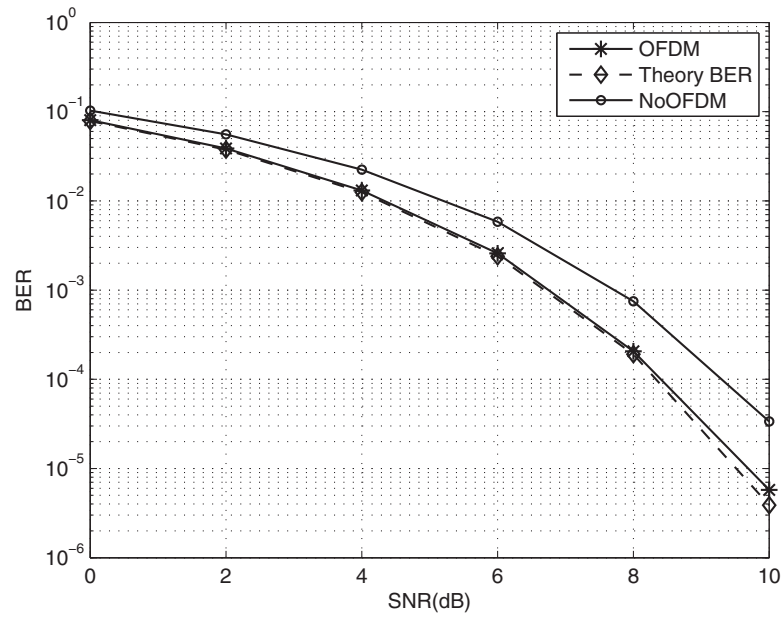

Fig. 7. BER curve for BPSK modulated signal using OFDM in AWGN channel

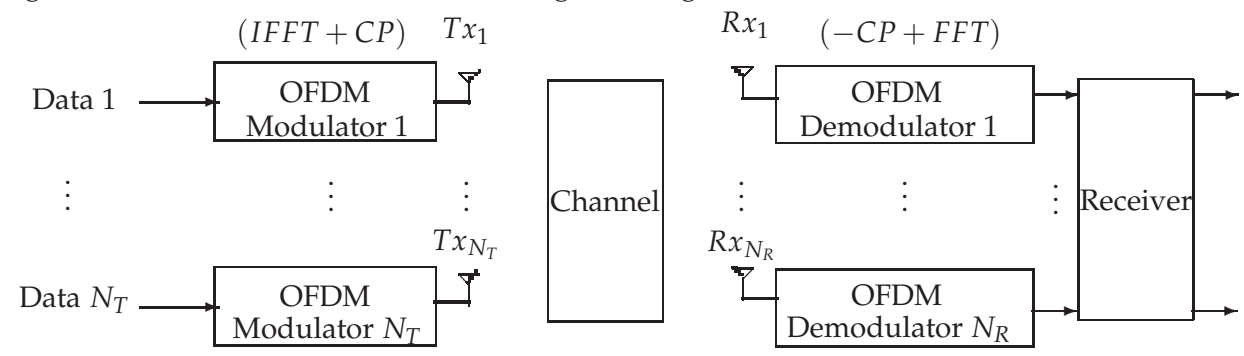

Fig. 8. MIMO-OFDM system

\subsection{Pattern diversity}

Pattern diversity consists of the use of several colocated antennas with different radiation patterns. This type of diversity can provide a higher gain versus a single omnidirectional antenna if antennas are enough spaced and adequately polarized.

\subsection{Polarization diversity}

Polarization diversity is a diversity technique where different polarizations are used. Horizontal and vertical polarizations could be used so that to provide diversity. At the MIMO receiver for example, the antennas take advantage of the multipath propagation characteristics to receive separate uncorrelated signals.

\section{MIMO channel modeling}

\subsection{Geometry-based Stochastic Channel Models (GSCM)}

Geometry-based Stochastic Channel Models(GSCM) have an immediate relation to physical reality. Such models are based on geometrical considerations, mainly scatterer locations and channel impulse response behavior. We distinguish the Double Bounce Geometry-based 
Stochastic Channel Models (DB-GSCM) and the Single Bounce Geometry-based Stochastic Channel Models (SB-GSCM).

\subsubsection{Double Bounce Geometry-based Stochastic Channel Models (DB-GSCM)}

Geometry based stochastic channel models represent the channel in a propagation-based stochastic way in which the geometry is represented by statistical means. The GSCM is based on the concept of clusters of scatterers around the transmitter and the receiver. The scatterer locations are defined according to a random fashion that follows a particular probability distribution. Scatterers represent discrete channel paths and can involve statistical characterizations of several propagation parameters such as delay spread, angular spread, spatial correlation and cross polarization discrimination. These parameters will be detailed in the following sections. Fig. 9 shows a random geometrical two circle model in which the geometry of the scatterers follows a circular distribution. Each propagation path is able to have two times of reflection by scatterers, one at transmitter side and another one at receiver side. Local scatterers around the transmit antennas and receive antennas are respectively situated in a circle of radius $R_{T}$ and a circle of radius $R_{R}$. The distance between the receive antennas and transmit antennas $\mathrm{D}$ is assumed to be longer than the radii $R_{T}$ and $R_{R}$ as depicted on Fig. 9.

Scatterers around the transmitter Scatterers around the receiver

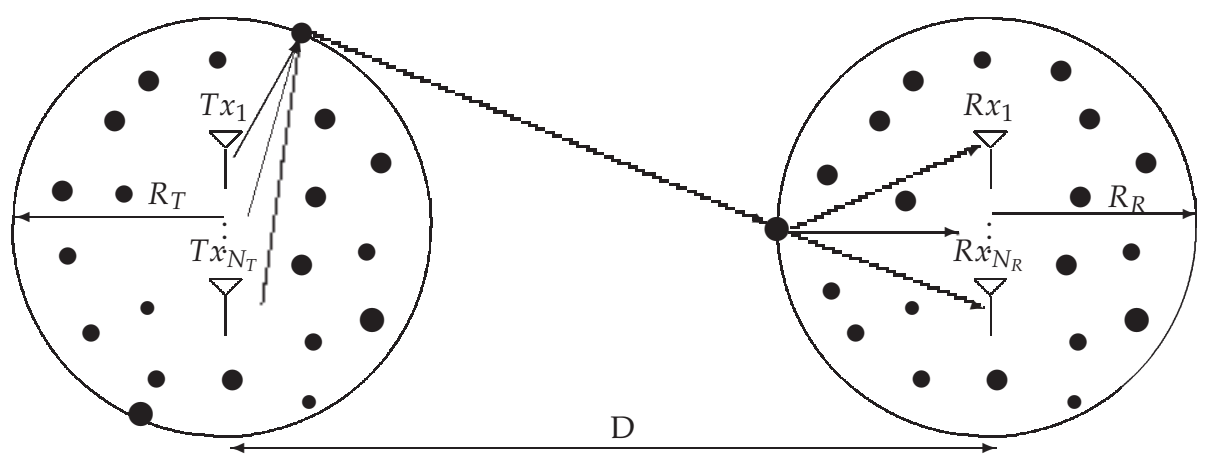

Fig. 9. Double Bounce Scattering Mechanism

\subsubsection{Single Bounce Geometry-based Stochastic Channel Models (SB-GSCM)}

When a single bounce of scatters is placed around the transmit antennas or the receive antennas, this is referred as SB-GSCM. SB-GSCM models are originally considered in systems where the base station is elevated and there is no local scattering obstruct while the mobile station (at the receive side) is surrounded by scatterers (Raoof \& Zhou, 2009).

\subsection{Stochastic channel models}

Stochastic channel models can be split into three categories :

1. Correlation based models

2. Stochastic models of scatterers

3. Based propagation models

In the following, we briefly review the listed channel models. 


\subsubsection{Correlation models}

\section{The independent and identically distributed (i.i.d) model}

The listed models are normally calculated analytically. Hence, the channel matrix $\mathbf{H}$ can be explicitly expressed. The simplest model is the i.i.d model. This supposes that multipath channels in presence of scatterers are independent and uniformly distributed in all directions. The MIMO channel coefficients are statically independent with equal variance.

\section{The Kronecker MIMO channel model}

Kronecker model assumes that spatial transmit correlation and spatial receive correlation are separable. Therefore, the full channel correlation matrix can be modeled by the Kronecker product of the transmit and receive correlation matrix. Full channel correlation matrices is expressed as :

$$
\text { Hcorr }=\mathbf{R}_{T x} \otimes \mathbf{R}_{R x}
$$

where :

- $\mathbf{R}_{T x}=\mathrm{E}\left[\mathbf{H}_{\mathrm{Kron}}^{H} \mathbf{H}_{\mathrm{Kron}}\right]$ is the transmit correlation matrix.

- $\mathbf{R}_{R x}=\mathrm{E}\left[\mathbf{H}_{\mathrm{Kron}} \mathbf{H}_{\mathrm{Kron}}^{H}\right]$ is the receive correlation matrix.

- $\otimes$ denotes the Kronecker product. The Kronecker product for matrices $\mathbf{A}$ and $\mathbf{C}$ is defined as :

$$
\mathbf{A} \otimes \mathbf{C}=\left(\begin{array}{cccc}
\mathbf{A}_{11} \mathbf{C} & \mathbf{A}_{12} \mathbf{C} & \ldots \\
\mathbf{A}_{21} \mathbf{C} & \mathbf{A}_{22} \mathbf{C} & \ldots \\
\ldots & \ldots & \ldots
\end{array}\right)
$$

The channel matrix according to the Kronecker model is expressed as (Biglieri et al., 2007):

$$
\mathbf{H}_{\mathrm{Kron}}=\mathbf{R}_{R x}^{1 / 2} \mathbf{H}_{w}\left(\mathbf{R}_{T x}^{1 / 2}\right)
$$

$\mathbf{H}_{w}$ is an i.i.d. Rayleigh fading channel. Note that there are others MIMO channel models based on the Kronecker such as the Keyhole model and Weichselberger model. A review of these models is presented in (Raoof \& Zhou, 2009).

\subsubsection{Stochastic scatterer model}

This section gives a generic description of stochastic models of scatterers. Multipath channels are grouped into clusters according to statistical considerations. Besides, parameters for the channel impulse response are determined in a random manner without referring to the geometry of a physical medium. We mainly focus on the Saleh \& Valenzuela (SVA) model (Saleh \& Valenzuela, 1987). For finite numbers of clusters and multipath components, the impulse response of the SVA channel model is expressed as :

$$
h(t)=\sum_{l=1}^{L c} \sum_{k=1}^{K_{m}} \alpha_{k, l} \exp \left(j \Psi_{k, l}\right) \delta\left(t-T_{l}-\tau_{k, l}\right)
$$

where :

- $L_{c}$ : Number of clusters which is Poisson distributed

- $K_{m}$ : Number of multipath propagation components which are grouped into a cluster

- $\alpha_{k, l}$ : Tap weight of the $k$-th path component of the $l$-th cluster

- $\Psi_{k, l}$ : The phase of the $k$-th path component of the $l$-th cluster 
- $T_{l}$ : Delay of the $l$-th cluster

- $\tau_{k, l}$ : Delay of the $k$-th multipath component relative to the $l$-th cluster arrival time $T_{l}$

- $\delta($.$) is the Dirac delta function$

We assume that $\tau_{k, l}, \quad k=1, \ldots, K_{m} ; \quad l=1, \ldots, L_{c}$ are computed relatively to the first propagation component. Therefore, $\tau_{1, l}=0 ; \quad l=1, \ldots, L_{\mathcal{c}}$. Both cluster delay and multipath component delay are given by Poisson processes. The described model was also extended to the spatial domain by including direction of departure and direction of arrival. The normalized directional channel impulse response can be written as :

$$
\begin{gathered}
h\left(t, \phi_{\mathrm{T}}, \phi_{\mathrm{R}}\right)=\frac{1}{\sqrt{L_{c} K_{m}}} \sum_{l=1}^{L_{c}} \sum_{k=1}^{K_{m}} \alpha_{k l} \exp \left(j \Psi_{k, l}\right) \delta\left(t-T_{l}-\tau_{k, l}\right) \\
\times \delta\left(\phi_{\mathrm{T}}-\Phi_{\mathrm{T}, l}-\phi_{\mathrm{T}, k, l}\right) \delta\left(\phi_{\mathrm{R}}-\Phi_{\mathrm{R}, l}-\phi_{\mathrm{R}, k, l}\right)
\end{gathered}
$$

here :

- $T_{l}$ : Initial arrival time

- $\Phi_{\mathrm{T}, l}$ : Mean departure angle of the $l$-th cluster

- $\Phi_{\mathrm{R}, l}$ : Mean arrival angle of the $l$-th cluster

- $\tau_{k, l}$ : Initial arrival time with respect to the $l$-th cluster

- $\phi_{T, k, l}$ : Departure angle with respect to the initial time and mean angle of the $l$-th cluster

- $\phi_{\mathrm{R}, k, l}$ : Arrival angle with respect to the initial time and mean angle of the $l$-th cluster

The parameters $L_{c}$ and $K_{m}$ are important for channel modeling design. They respectively depend on two other parameters which are the cluster decay factor and the ray decay factor. Fig. 10 shows the simulation results for the SVA model channel where four clusters of multipath components are obtained.

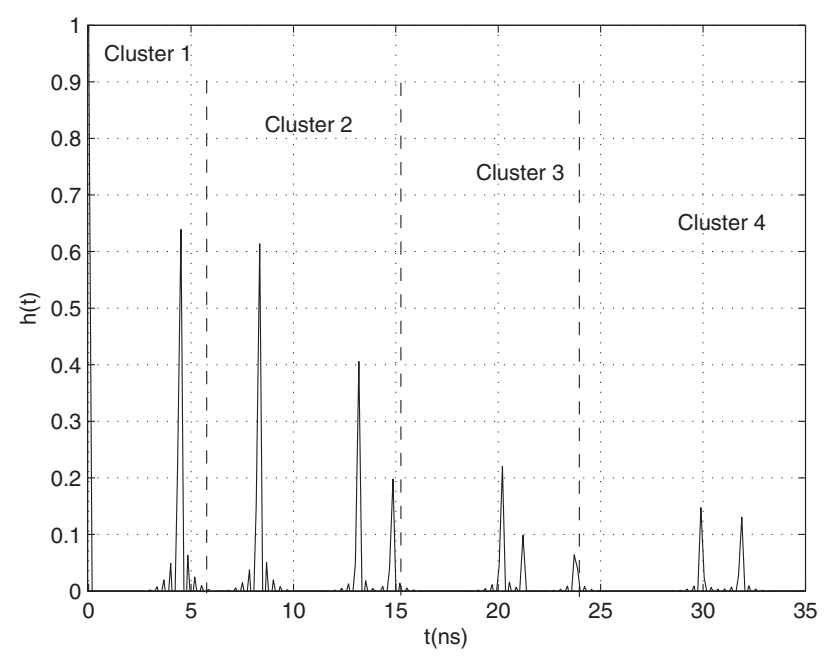

Fig. 10. Saleh \& Valenzuela channel impulse response model for a SISO link 


\subsubsection{Geometrical propagation model}

Several existing analytic models are not coinciding with real situation. In fact, they rarely consider the effect of topology structure on radio channel propagation. Geometrical-based propagation model shows a more general model in which propagation considerations are involved. For seek of brevity, we consider the example of the finite scatterer model. Let us consider the Uniform Linear Antenna (ULA) antennas at both the transmit and the receive sides. Assume that the element spacing between two close antennas at transmit and receive sides are respectively denoted by $d_{T}$ and $d_{R}$. We consider finite number of scatterers that are located far away from the transmitter and the receiver. In the finite scatterer model, each path specifies a Direction of Departure (DOD) $\phi_{\mathrm{T}}$ from the transmitting array and the Direction of Arrival (DOA) $\phi_{\mathrm{R}}$ at the receiving array. According to these considerations, transmitting and receiving steering vectors are expressed as (Burr, 2003):

$$
\begin{aligned}
& \mathbf{a}_{\mathrm{T}}\left(\theta_{\mathrm{T}}\right)=\left[1, \exp \left\{-j 2 \pi \theta_{\mathrm{T}}\right\}, \cdots, \exp \left\{-j 2 \pi\left(N_{\mathrm{T}}-1\right) \theta_{\mathrm{T}}\right\}\right]^{T} \\
& \mathbf{a}_{\mathrm{R}}\left(\theta_{\mathrm{R}}\right)=\left[1, \exp \left\{-j 2 \pi \theta_{\mathrm{R}}\right\}, \cdots, \exp \left\{-j 2 \pi\left(N_{\mathrm{R}}-1\right) \theta_{\mathrm{R}}\right\}\right]^{T}
\end{aligned}
$$

- $\theta_{\mathrm{T}}=d_{\mathrm{T}} \sin \left(\phi_{\mathrm{T}}\right) / \lambda$

- $\theta_{\mathrm{R}}=d_{R} \sin \left(\phi_{\mathrm{R}}\right) / \lambda$

- $\lambda$ is the wavelength of radio propagation.

The discrete channel model with $L_{s}$ scatterers is therefore expressed via the array steering and response vectors as:

$$
\mathbf{H}_{\mathbf{S}}=\sum_{l=1}^{L_{\mathrm{s}}} \beta_{l} \mathbf{a}_{\mathrm{R}}\left(\theta_{\mathrm{R}, l}\right) \mathbf{a}_{\mathrm{T}}^{H}\left(\theta_{\mathrm{T}, l}\right)=\mathbf{A}_{\mathrm{R}}\left(\theta_{\mathrm{R}}\right) \mathbf{H}_{\mathrm{P}} \mathbf{A}_{\mathrm{T}}^{H}\left(\theta_{\mathrm{T}}\right)
$$

- $\beta_{l}$ is the complex amplitude of the $l$-th path

- $\mathbf{H}_{\mathrm{P}}=\operatorname{diag}\left(\beta_{1}, \cdots, \beta_{L_{s}}\right)$

- $\mathbf{A}_{\mathrm{T}}\left(\theta_{\mathrm{T}, l}\right)=\left[\mathbf{a}_{\mathrm{T}}\left(\theta_{\mathrm{T}, 1}\right), \cdots, \mathbf{a}_{\mathrm{T}}\left(\theta_{\mathrm{T}, L_{\mathrm{S}}}\right)\right]$

- $\mathbf{A}_{\mathrm{R}}\left(\theta_{\mathrm{R}, l}\right)=\left[\mathbf{a}_{\mathrm{R}}\left(\theta_{\mathrm{R}, 1}\right), \cdots, \mathbf{a}_{\mathrm{R}}\left(\theta_{\mathrm{R}, L_{s}}\right)\right]$

\section{Performance analysis of MIMO systems based on capacity}

\subsection{Some entropy terminologies (G.Proakis, 1995)}

We briefly review in this paragraph some terminologies that we need for the channel capacity derivation.

Entropy: The entropy $\mathcal{H}(X)$ of a variable $X$ measures the uncertainty about the realization of $X$. Let $X$ be a random variable with a probability function $p(x)=P\{X=x\}, x=x_{1}, \ldots, x_{n}$ are possible values of $X$ from a set of possible realizations $\chi$. The entropy $\mathcal{H}(X)$ of the variable $X$ is expressed as :

$$
\begin{aligned}
\mathcal{H}(X) & =\mathrm{E}\left[-\log _{2}(p(x))\right] \\
& =-\sum_{x \in \chi} p(x) \log _{2}(p(x))
\end{aligned}
$$

E denotes the expected function. 
Joint entropy: The joint entropy measures how much information is contained in a joint system of two random variables. Given two random variables $X$ and $Y$ with respective probability functions $p(x)$ and $p(y)$, the joint entropy is expressed as :

$$
\mathcal{H}(X, Y)=-\sum_{x \in \chi} \sum_{y \in Y} p(x, y) \log _{2}(p(x, y))
$$

$Y$ denotes the set of possible values of $y$.

Conditional entropy : Suppose $X$ and $Y$ are random variables. Then, for any fixed value $x$ of $X$, we get a conditional probability distribution on $Y$. We denote the associated random variable by $\mathcal{H}(Y \mid X)$. Conditional entropy is then :

$$
\mathcal{H}(Y \mid X)=\mathcal{H}(X, Y)-H(X)
$$

Mutual information: Mutual information is a quantity that measures the dependence between two arbitrary random variables. The mutual information between two discrete random variables $X$ and $Y$ is defined to be :

$$
\begin{aligned}
\mathcal{I}(X, Y) & =\mathcal{H}(X)+\mathcal{H}(Y)-\mathcal{H}(X, Y) \\
& =\mathcal{H}(Y)-\mathcal{H}(Y \mid X)
\end{aligned}
$$

\subsection{Capacity definition based on information theory} Deterministic capacity

Channel capacity measures the maximum amount of information that could be transmitted through a channel and received with negligible error. Hence :

$$
C=\max _{p(x)} \mathcal{I}(X, Y)
$$

For a SISO link with input signal $x$, AWGN $b$ and a constant channel gain $h$, the output signal $y$ is expressed as :

$$
y=h x+b
$$

The mutual information is expressed as :

$$
\begin{aligned}
\mathcal{I}(x, y) & =\mathcal{H}(y)-\mathcal{H}(b) \\
& \leq \log _{2}\left(\pi e\left(P_{T}+\sigma_{b}^{2}\right)\right)-\log _{2}\left(\pi e \sigma_{b}^{2}\right) \\
& =\log _{2}\left(1+\frac{P_{T}}{\sigma_{b}^{2}}\right)
\end{aligned}
$$

where :

- $P_{T}=\mathrm{E}\left\{|\mathrm{x}|^{2}\right\}$ is the transmit power

- $\sigma_{b}^{2}=\mathrm{E}\left\{|\mathrm{b}|^{2}\right\}$ is the noise power

- $\log (e)=1$

Finally, the normalized channel capacity is given by :

$$
C_{\text {SISO }}=\log _{2}\left(1+\frac{P_{T}}{\sigma_{b}^{2}}\right) \quad \text { bits } / \mathrm{s} / \mathrm{Hz}
$$

This is referred as the famous Shannon's channel capacity. 


\section{Ergodic capacity}

In information-theoretical sense, ergodic capacity refers to the maximum rate that communication can be achieved; assuming that the communication duration is long enough to exploit all channel state. In fact, the propagation channel varies in time. This causes the channel capacity varying in time. Consequently, to measure the fluctuating channel capacity in information theory, ergodic capacity is defined. Ergodic capacity refers to the maximum rate that can be achieved during a long observation communication to exploit all channel information. Ergodic capacity $\bar{C}_{\text {SISO }}$ is an expected value. For a SISO channel $h, \bar{C}_{\text {SISO }}$ is derived as :

$$
\bar{C}_{\text {SISO }}=\mathrm{E}\left\{\max _{p(x): \mathrm{E}\left\{|\mathrm{x}|^{2}\right\} \leqslant \mathrm{P}_{\mathrm{T}}} \mathcal{I}(\mathrm{x}, \mathrm{y})\right\} \quad \text { bits } / \mathrm{s} / \mathrm{Hz}
$$

which could be also expressed as :

$$
\overline{\mathrm{C}}_{\mathrm{SISO}}=\mathrm{E}\left\{\log _{2}\left(1+\frac{P_{T}}{\sigma_{b}^{2}}|h|^{2}\right)\right\} \quad \text { bits } / \mathrm{s} / \mathrm{Hz}
$$

\section{Outage capacity}

Outage capacity is an another statical parameter on which relies the channel performance. Outage capacity is defined as the probability that the capacity $C(h)$ is lower than a certain threshold $C_{\text {out }}$. Outage capacity is expressed as :

$$
P_{\text {out }}=\operatorname{Pr}\left(C(h)<C_{\text {out }}\right)
$$

Outage probability is related to the Complementary Cumulative Distribution Function (CCDF):

$$
\mathrm{CCDF}=1-P_{\text {out }}
$$

In the following, we consider a MIMO system with $N_{T}$ transmit antennas and $N_{R}$ receive antennas. We assume that the channel is flat fading. The received signal at antenna $q, y_{q}$ is expressed as :

$$
y_{q}=\sum_{p=1}^{N_{T}} h_{q p} x_{p}+b_{q} \quad ; q=1, \ldots, N_{R}
$$

The MIMO channel capacity is derived as :

$$
\mathcal{I}(\mathbf{x}, \mathbf{y})=\mathcal{H}(\mathbf{y})-\mathcal{H}(\mathbf{y} \mid \mathbf{x})
$$

$\mathcal{H}(\mathbf{y})$ and $\mathcal{H}(\mathbf{y} \mid \mathbf{x})$ are respectively the received signal entropy and the entropy of $\mathbf{y} \mid \mathbf{x}$. As the received signal $\mathbf{y}$ and the signal noise $\mathbf{b}$ are independents, $\mathcal{H}(\mathbf{y} \mid \mathbf{x})=\mathcal{H}(\mathbf{b})$. Thereafter, the capacity is obtained by maximizing the received signal entropy. For MIMO capacity derivation, we denote :

- $\mathbf{R}_{\mathbf{x}}=\mathrm{E}\left\{\mathbf{x x}^{H}\right\}$ : Covariance matrix of the transmit signal

- $\mathbf{R}_{\mathbf{b}}=\mathrm{E}\left\{\mathbf{b b}^{H}\right\}$ : Covariance matrix of the noise signal

- $\mathbf{R}_{\mathbf{y}}=\mathrm{E}\left\{\mathbf{y} \mathbf{y}^{H}\right\}:$ Covariance matrix of the received signal 
Thus :

$$
\mathbf{R}_{\mathbf{y}}=\mathbf{H} \mathbf{R}_{\mathbf{x}} \mathbf{H}^{H}+\mathbf{R}_{\mathbf{b}}
$$

The mutual information is expressed as :

$$
\begin{aligned}
\mathcal{I}(\mathbf{x}, \mathbf{y} \mid \mathbf{H}) & =\log _{2} \operatorname{det}\left(\pi e \mathbf{R}_{\mathbf{y}}\right)-\log _{2} \operatorname{det}\left(\pi e \mathbf{R}_{\mathbf{b}}\right) \\
& =\log _{2} \operatorname{det}\left\{\mathbf{I}_{N_{R}}+\mathbf{H R}_{\mathbf{x}} \mathbf{H}^{H}\left(\mathbf{R}_{\mathbf{b}}\right)^{-\mathbf{1}}\right\}
\end{aligned}
$$

For circularly symmetric Gaussian random vectors, the mutual information is maximum and also expressed as :

$$
C_{\mathrm{MIMO}}=\max _{p(\mathbf{x}): \mathrm{E}\left\{\mathbf{x}^{\mathrm{H}} \mathbf{x}\right\} \leq \mathrm{P}_{\mathrm{T}}} \mathcal{I}(\mathbf{x}, \mathbf{y} \mid \mathbf{H}) \quad \text { bits } / \mathrm{s} / \mathrm{Hz}
$$

When no CSI (Channel State Information) is available at the transmitter, equal power allocation is adopted. With the assumption that no correlation exits at the transmit side,

$$
\mathbf{R}_{\mathbf{x}}=\frac{P_{T}}{N_{T}} \mathbf{I}_{N_{T}}
$$

$P_{T}$ is the total power available at the transmit side.

The MIMO capacity is then expressed as :

$$
C_{\mathrm{MIMO}}=\log _{2} \operatorname{det}\left(\mathbf{I}_{N_{R}}+\frac{\gamma}{N_{T}} \mathbf{H H}^{H}\right) \quad \text { bits } / \mathrm{s} / \mathrm{Hz}
$$

$\gamma$ is the SNR.

\subsection{MIMO capacity based on SVD: CSI known at the receiver}

When CSI is available at the receiver, SVD factorization is used and MIMO channel capacity could be easily derived. Let us first review the SVD technique. SVD is a factorization method for complex matrix which is widely used in signal processing. We take an $(N \times M)$ matrix A, SVD theorem states:

$$
\mathbf{A}=\mathbf{U S V}^{H}
$$

- The eigenvectors of $\mathbf{A} \mathbf{A}^{H}$ make up the columns of $\mathbf{U}(N \times N)$ which is an unitary matrix $\left(\mathbf{U U}^{H}=\mathbf{I}_{N}\right)$.

- The singular values in $\mathbf{S}(N \times M)$ are square roots of eigenvalues from $\mathbf{A A}^{H}$ or $\mathbf{A}^{H} \mathbf{A}$. The singular values are the diagonal entries of the $\mathbf{S}$ matrix and are arranged in descending order.

- The eigenvectors of $\mathbf{A}^{H} \mathbf{A}$ make up the columns of $\mathbf{V} . \mathbf{V}(M \times M)$ is also a unitary matrix $\left(\mathbf{V V}^{H}=\mathbf{I}_{M}\right)$.

Calculating the SVD of the MIMO channel matrix $\mathbf{H}$ leads to the following factorization :

$$
\mathbf{H}=\mathbf{U S V}^{H}
$$

We substitute $\mathbf{H}$ by its SVD decomposition. Hence, the received signal is expressed as :

$$
\mathbf{y}=\mathbf{U S} \mathbf{V}^{H} \mathbf{x}+\mathbf{b}
$$


Let :

$$
\begin{aligned}
\mathbf{y}^{\prime} & =\mathbf{U}^{H} \mathbf{y} \\
\mathbf{x}^{\prime} & =\mathbf{V}^{H} \mathbf{x} \\
\mathbf{b}^{\prime} & =\mathbf{U}^{H} \mathbf{b}
\end{aligned}
$$

As $\mathbf{U}$ and $\mathbf{V}$ are unitary matrix, variables $\mathbf{x}^{\prime}$ and $\mathbf{b}^{\prime}$ keep the same statistical densities as $\mathbf{x}$ and b. Therefore, the channel model $(\mathbf{y}=\mathbf{H x}+\mathbf{b})$ could be also presented as :

$$
\mathbf{y}^{\prime}=\mathbf{S} \mathbf{x}^{\prime}+\mathbf{b}^{\prime}
$$

- $\mathbf{y}^{\prime}=\left[y_{1}^{\prime}, \ldots, y_{N_{R}}^{\prime}\right]^{T}$

- $\mathbf{x}^{\prime}=\left[x_{1}^{\prime}, \ldots, x_{N_{T}}^{\prime}\right]^{T}$

- $\mathbf{S}=\operatorname{diag}\left(\sqrt{\lambda_{1}}, \ldots, \sqrt{\lambda_{R}}, 0, \ldots, 0\right) \quad ; R=\min \left(N_{R}, N_{T}\right)$ is the rank of the channel matrix $\mathbf{H}$.

Equation (1) can be rewritten as:

$$
\mathbf{y}_{i}^{\prime}= \begin{cases}\sqrt{\lambda_{i}} x_{i}^{\prime}+b_{i}^{\prime}, & \mathrm{i}=1, \ldots, R ; \\ b_{i}^{\prime}, & i=R+1, \ldots, N_{R} .\end{cases}
$$

According to the equation above, the MIMO channel consists of $R$ uncorrelated subchannels. The covariance matrix of the signals $\mathbf{y}^{\prime}, \mathbf{x}^{\prime}$ and $\mathbf{b}^{\prime}$ are expressed as :

$$
\begin{aligned}
\mathbf{R}_{\mathbf{y}^{\prime} \mathbf{y}^{\prime}} & =U^{H} \mathbf{R}_{\mathbf{y y}} \mathbf{U} \\
\mathbf{R}_{\mathbf{x}^{\prime} \mathbf{x}^{\prime}} & =V^{H} \mathbf{R}_{\mathbf{x x}} \mathbf{V} \\
\mathbf{R}_{\mathbf{b}^{\prime} \mathbf{b}^{\prime}} & =U^{H} \mathbf{R}_{\mathbf{b} \mathbf{b}} \mathbf{U}
\end{aligned}
$$

and

$$
\begin{aligned}
\operatorname{tr}\left(\mathbf{R}_{\mathbf{y}^{\prime} \mathbf{y}^{\prime}}\right) & =\operatorname{tr}\left(\mathbf{R}_{\mathbf{y} \mathbf{y}}\right) \\
\operatorname{tr}\left(\mathbf{R}_{\mathbf{x}^{\prime} \mathbf{x}^{\prime}}\right) & =\operatorname{tr}\left(\mathbf{R}_{\mathbf{x} \mathbf{x}}\right) \\
\operatorname{tr}\left(\mathbf{R}_{\mathbf{b}^{\prime} \mathbf{b}^{\prime}}\right) & =\operatorname{tr}\left(\mathbf{R}_{\mathbf{b} \mathbf{b}}\right)
\end{aligned}
$$

The capacity of the MIMO channel is the summation of the $R$ uncorrelated subchannels. Hence:

$$
\begin{aligned}
C_{\text {SVD }} & =\sum_{i=1}^{R} \log _{2}\left(1+\frac{\gamma \cdot \lambda_{i}}{N_{T}}\right) \\
& =\log _{2} \prod_{i=1}^{R}\left(1+\frac{\gamma \cdot \lambda_{i}}{N_{T}}\right) \quad ; \gamma=\frac{P_{T}}{\sigma_{b}^{2}} \quad \text { bits } / \mathrm{s} / \mathrm{Hz}
\end{aligned}
$$

One eigenvalue $\lambda$ of $\mathbf{H H}^{H}$ is obtained according to the following equation :

$$
\left(\lambda \mathbf{I}_{R}-\mathbf{Q}\right) \mathbf{y}=0 \quad ; \mathbf{y} \neq 0
$$


$\mathbf{Q}$ is the Wishart matrix :

$$
\mathbf{Q}=\left\{\begin{array}{l}
\mathbf{H H}^{H}, N_{R}<N_{T} \\
\mathbf{H}^{H} \mathbf{H}, N_{R} \geq N_{T}
\end{array}\right.
$$

$\lambda$ is an eigenvalue of the matrix channel $\mathbf{H}$. Hence :

$$
\operatorname{det}\left(\lambda \mathbf{I}_{R}-\mathbf{Q}\right)=\mathbf{0}
$$

The associate characteristic polynomial of the channel matrix is the polynomial defined by :

$$
p(\lambda)=\operatorname{det}\left(\lambda \mathbf{I}_{R}-\mathbf{Q}\right)
$$

which is also expressed as :

$$
p(\lambda)=\prod_{i=1}^{R}\left(\lambda-\lambda_{i}\right)
$$

If we substitute $\lambda$ by $\left(-N_{T} \frac{\sigma_{b}^{2}}{P_{T}}\right)$ then :

$$
\prod_{i=1}^{R}\left(1+\frac{\gamma \cdot \lambda_{i}}{N_{T}}\right)=\operatorname{det}\left(\mathbf{I}_{R}+\frac{\mathbf{f l}}{\mathbf{N}_{\mathbf{T}}} \mathbf{Q}\right) \quad ; \gamma=\frac{P_{T}}{\sigma_{b}^{2}}
$$

If $N_{R}<N_{T}$ then equation (43) becomes :

$$
C_{\mathrm{SVD}}=\log _{2} \operatorname{det}\left(\mathbf{I}_{R}+\frac{\gamma}{N_{T}} \mathbf{H H}^{H}\right) \quad \text { bits } / \mathrm{s} / \mathrm{Hz}
$$

Finally,

$$
C_{\mathrm{SVD}}=R \cdot \log _{2} \operatorname{det}\left(1+\frac{\gamma}{N_{T}} \mathbf{H H}^{\mathbf{H}}\right) \quad \text { bits } / \mathrm{s} / \mathrm{Hz}
$$

Simulation results for the ergodic MIMO capacity when CSI is available at the receiver is depicted in the Fig. 11. For a MIMO system with two transmit antennas, ergodic capacity increases linearly with the number of antennas. Ergodic capacity depends on the SNR level. Plotted curves show that capacity grows with the SNR.

\subsection{MIMO capacity based on Water-filling technique : CSI known at both transmitting and receiving sides}

When CSI is available at both the transmitter and the receiver, an optimal power allocation could be exploited. This is referred as the water-filling technique. The main idea of water-filling strategy is to allocate more power to better subchannels with higher SNR so as to maximize the sum of data rates in all subchannels where in each subchannel the data rate is related to the power allocation by Shannon's Gaussian capacity formula $\frac{1}{2} \log _{2}(1+\mathrm{SNR})$. 


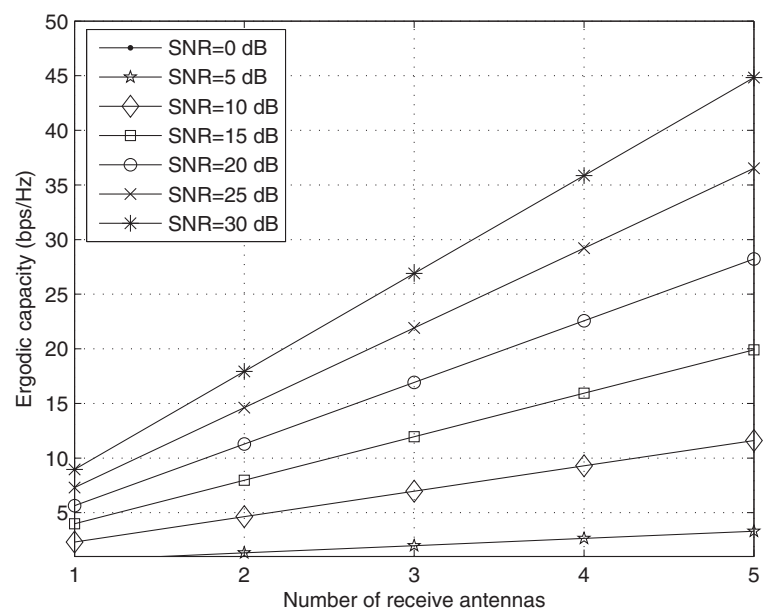

Fig. 11. Ergodic capacity derived by SVD

\section{Water-filling strategy}

The concept of the Water-filling technique (Yu \& Cioffi, 2001) relies on the maximization of the MIMO channel capacity under a total transmit power constraint.

$$
\sum_{p=1}^{N_{T}} P_{p}=P_{T} \quad ; p=1, \ldots, N_{T}
$$

The channel capacity is given by :

$$
C_{\mathrm{WF}}=\sum_{p=1}^{N_{T}} \log _{2}\left(1+\frac{P_{p}}{\sigma_{b}^{2}} \lambda_{p}\right) \quad \text { bits } / \mathrm{s} / \mathrm{Hz}
$$

Let :

$$
\mathbf{Z}=\sum_{p=1}^{N_{T}} \log _{2}\left(1+\frac{P_{p}}{\sigma_{b}^{2}} \lambda_{p}\right)+\mathbf{L}\left(P_{T}-\sum_{p=1}^{N_{T}} P_{p}\right)
$$

- $\mathbf{L}$ is the Lagrangian

- $\lambda_{p}$ is the $p$-th singular value of the channel

- $\sigma_{b}^{2}$ is the noise signal variance

Allocated powers $P_{p} ; p=1, \ldots, N_{T}$ are obtained by solving :

$$
\frac{\partial \mathbf{Z}}{\partial P_{p}}=0 \quad ; p=1, \ldots, N_{T}
$$

Hence,

$$
P_{p}=\mu-\frac{\sigma_{b}^{2}}{\lambda_{p}}
$$

$\mu$ is a constant scalar that satisfies the total power constraint (See equation (50)). 


\section{Capacity calculation based on Water-filling}

Let $R$ be the rank of $\mathbf{H}$. The allocated power for the subchannel $p$ is expressed as :

$$
P_{p}=\left(\mu-\frac{\sigma_{b}^{2}}{\lambda_{p}}\right)^{+} ; p=1, \ldots, R
$$

where :

$$
a^{+}=\max (a, 0)
$$

The received power at the subchannel $p$ is then :

$$
P_{r p}=\left(\lambda_{p} \mu-\sigma_{b}^{2}\right)^{+}
$$

Hence, the channel capacity is expressed as :

$$
C_{\mathrm{WF}}=\sum_{p} \log _{2}\left(1+\frac{P_{r p}}{\sigma_{b}^{2}}\right) \quad \text { bits } / \mathrm{s} / \mathrm{Hz}
$$

Finally, the channel capacity is :

$$
C_{\mathrm{WF}}=\sum_{p=1}^{R} \log _{2}\left[\left(\frac{\lambda_{p} \mu}{\sigma_{b}^{2}}\right)^{+}\right] \quad \text { bits } / \mathrm{s} / \mathrm{Hz}
$$

\section{Some numerical results for Water-Filling technique}

We present some numerical results in order to simulate the performance of the water-filling technique. Let us consider a correlated $\operatorname{MIMO}(4 \times 4)$ channel that follows the Kronecker model. We simulate the ergodic capacity according to both cases:

1. Equal power allocation for the transmit antennas

2. Optimal power allocation with Water-filling (WF) algorithm

Simulation results are depicted in Fig. 12. The MIMO capacity is improved by optimal power allocation strategy but stills affected by channel correlation. Simulation of the CCDF is shown in Fig. 13. The CCDF is improved by exploiting the WF technique for optimal power allocation. We present simulation results for the CCDF for two SNR values: SNR $=6 \mathrm{~dB}$ and $\mathrm{SNR}=10 \mathrm{~dB}$.

\section{Water-filling technique: discussion}

Water-filling provides an optimal power allocation and is an attractive strategy for capacity improvement. Nevertheless, capacity gain appears significant when more transmit antennas then receive antennas are deployed, i.e $N_{R} \leq N_{T}$. Moreover, this gain is considerable for low SNRs and is specially interesting in the case of correlated channels. Fig. 14 shows that the capacity gain is negligible for low SNRs and is almost null for high SNR values. CCDFs for the MIMO $(4 \times 2)$ are depicted in Fig. 15 and Fig. 16 respectively for high SNR value that is equal to $18 \mathrm{~dB}$ and low SNR of $2 \mathrm{~dB}$. Simulation results confirm that WF technique brings more performances for high noise strength and correlated MIMO channel. Finally, at high SNR value, the WF gain in ergodic capacity for $\operatorname{MIMO}\left(\mathrm{N}_{\mathrm{R}} \times \mathrm{N}_{\mathrm{T}}\right)$ is expressed as (Prayongpun, 2009):

$$
\bar{C}_{\mathrm{WF}}-\bar{C}_{\mathrm{MIMO}}=\left\{\begin{array}{lll}
0 & \text { if } & N_{T} \leq N_{R} \\
R \log _{2}\left(\frac{N_{T}}{R}\right) & \text { if } & N_{T}>N_{R}
\end{array}\right.
$$

where $R=\min \left(N_{R}, N_{T}\right)$. 


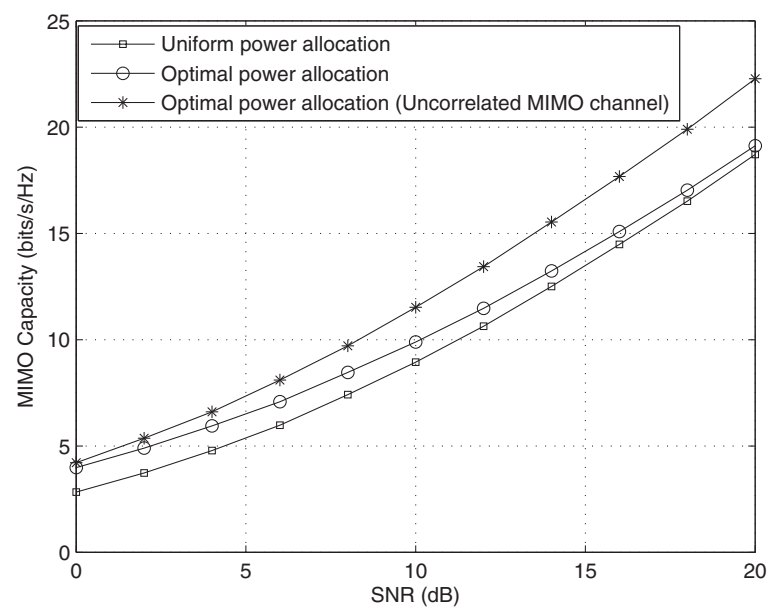

Fig. 12. $\mathrm{MIMO}(4 \times 4)$ : Capacity improvement with WF strategy-Channel correlation impact on system capacity

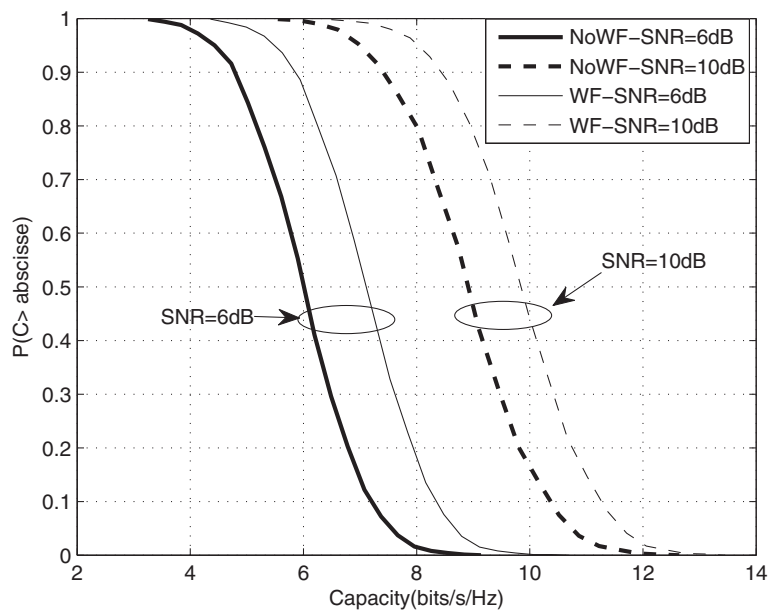

Fig. 13. CCDF for $\mathrm{MIMO}(4 \times 4)$ with various $\mathrm{SNR}$ values 


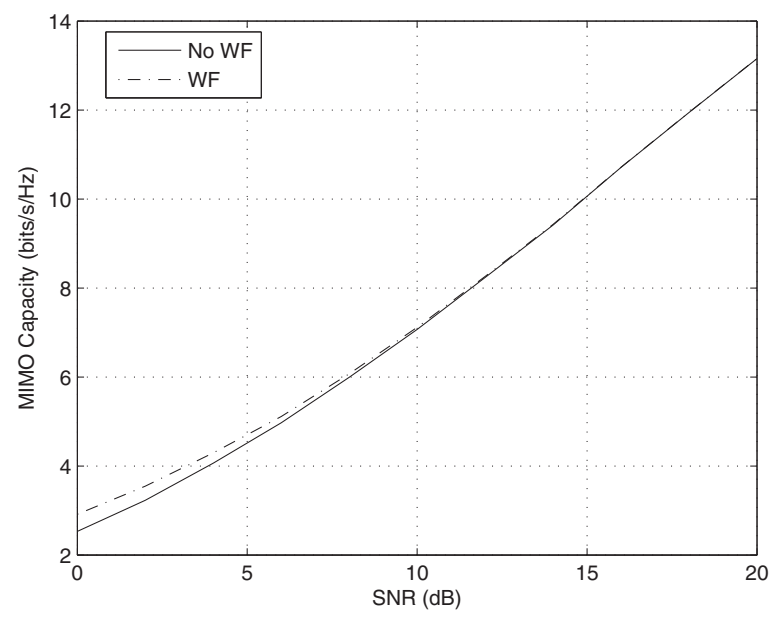

Fig. 14. Ergodic capacity for $\mathrm{MIMO}(4 \times 2)$-Kronecker channel model

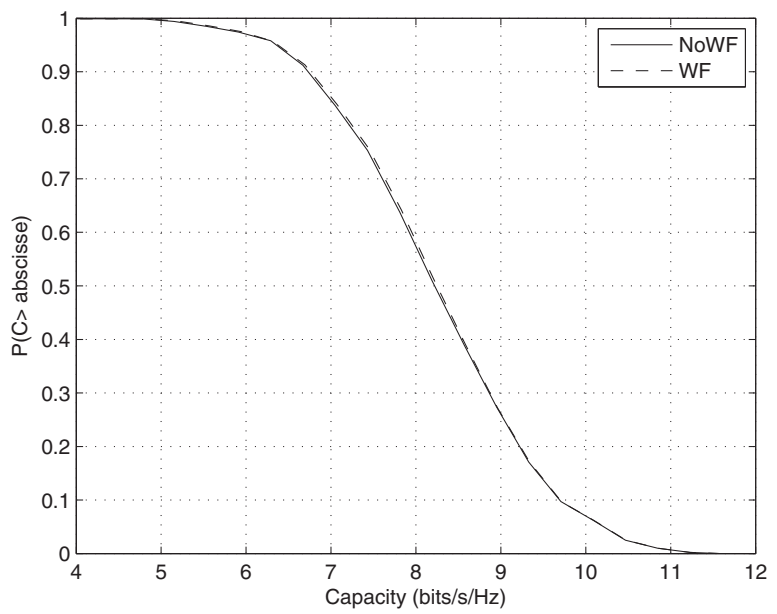

Fig. 15. CCDF for $\mathrm{MIMO}(4 \times 2)$-Kronecker channel model $(\mathrm{SNR}=18 \mathrm{~dB})$ 


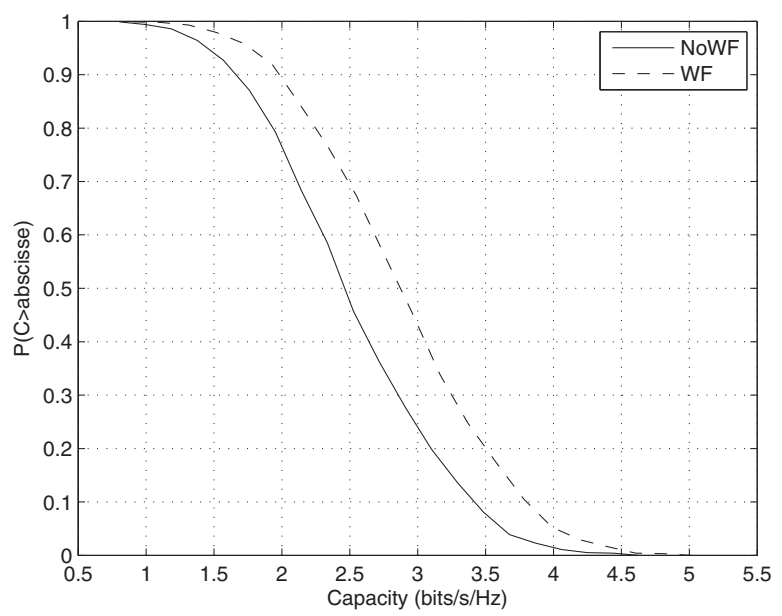

Fig. 16. $\mathrm{CCDF}$ for $\mathrm{MIMO}(4 \times 2)-$ Kronecker channel model $(\mathrm{SNR}=2 \mathrm{~dB})$

\section{Combining techniques for MIMO systems}

MIMO system can use several techniques at the receiver so that to combine the multiple incoming signals for more robust reception. Combining techniques are listed below :

1. Maximal Ratio Combining (MRC): Incoming signals are combined proportional to the SNR of that path signal. The MRC coefficients correspond to the relative amplitudes of the pulse replicas received by each antenna such that more emphasis is placed on stronger multipath components and less on weaker ones.

2. Equal Gain Combining (EGC) simply adds the path signals after they have been cophased (Sanayei \& Nosratinia, 2004).

3. Selection Combining (SC) selects the highest strength of incoming signals from one of the receiving antennas.

Combining techniques can be carried so that to satisfy one or more targets :

1. Maximizing the diversity gain

2. Maximizing the multiplexing gain

3. Achieving a compromise between diversity gain and multiplexing gain

4. Achieving best performances in terms of Bit Error Rate (BER)

5. Maximizing the Frobenius norm of the MIMO channel and therefore the MIMO channel capacity

Let us recall the SIMO system model with $N_{R}$ receive antennas. The received signal at the $q$-th receive antenna is expressed as :

$$
y_{q}=h_{q} x+b_{q} \quad ; q=1, \ldots, N_{R}
$$

$h_{q}$ is the $q$-th complex channel gain, $b_{q}$ is an AWGN with zero mean and variance $\sigma_{b}^{2}$.

We keep for notations : 
- $P_{T}$ : Transmit signal power

- $\gamma=\frac{P_{T}}{\sigma_{b}^{2}}$ is the SNR

We assume channel normalization and a perfect channel estimation. We will be more interested in the combining module. Our aim is to derive the combining coefficients $g_{q} ; q=$ $1, \ldots, N_{R}$. The output signal at the combining module can be expressed as:

$$
y=x \sum_{q=1}^{N_{R}} g_{q} h_{q}+\sum_{q=1}^{N_{R}} g_{q} b_{q}
$$

Combining technique in MIMO system is depicted in Fig.17. Combining coefficients relative to the listed techniques are given by:

\begin{tabular}{|l|l|}
\hline Combining technique & Combining coefficient \\
\hline \hline MRC & $g_{q}=h_{q}^{*}$ \\
\hline EGC & $g_{q}=\frac{h_{q}^{*}}{\left|h_{q}\right|}$ \\
\hline SC & $g_{q}=\left\{\begin{array}{l}1,\left|h_{q}\right| \geqslant\left|h_{k}\right|, \forall k \neq q ; \\
0, \text { otherwise. }\end{array}\right.$ \\
\hline
\end{tabular}

Table 1. Combining coefficients

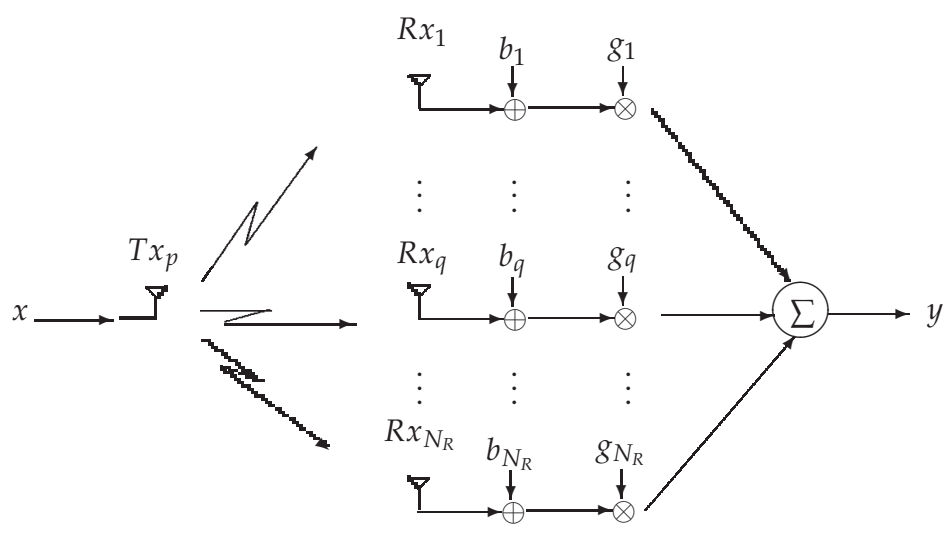

Fig. 17. SIMO system with combining technique

\subsection{Maximal Ratio Combining (MRC)}

The equivalent SNR of MRC has been calculated as :

$$
\gamma_{y}=\gamma \cdot \frac{\left(\sum_{q=1}^{N_{R}}\left|h_{q}\right|^{2}\right)^{2}}{\sum_{q=1}^{N_{R}}\left|h_{q}\right|^{2}}=\gamma \cdot \sum_{q=1}^{N_{R}}\left|h_{q}\right|^{2}=\sum_{q=1}^{N_{R}} \gamma_{q}
$$

Thus, the instantaneous SNR $\gamma_{y}$ is expressed as the sum of the instantaneous SNR at different receive antennas. For normalized channel matrix, the SNR is then:

$$
\gamma_{y}=N_{R} \cdot \gamma
$$


The system capacity with MRC is :

$$
C_{\mathrm{MRC}}=\log _{2}\left(1+\gamma \cdot \sum_{q=1}^{N_{R}}\left|h_{q}\right|^{2}\right) \quad \text { bits } / \mathrm{s} / \mathrm{Hz}
$$

\subsection{Equal Gain Combining (EGC)}

The instantaneous SNR is expressed as :

$$
\gamma_{y}=\frac{\gamma}{N_{R}} \cdot\left(\sum_{q=1}^{N_{R}}\left|h_{q}\right|\right)^{2}
$$

Resulting capacity has been calculated as :

$$
C_{\mathrm{EGC}}=\log _{2}\left(1+\frac{\gamma}{N_{R}} \cdot \sum_{q=1}^{N_{R}}\left|h_{q}\right|^{2}\right) \quad \text { bits } / \mathrm{s} / \mathrm{Hz}
$$

\subsection{Selection Combining(SC)}

The receiver scans the antennas, finds the antenna with the highest instantaneous SNR and selects it. We denote the highest received instantaneous SNR as :

$$
\gamma_{y}=\max \left(\gamma_{1}, \ldots, \gamma_{N_{R}}\right)
$$

The SNR at the output of the combiner for an uncorrelated channel is:

$$
\gamma_{y}=\gamma \cdot \sum_{q=1}^{N_{R}} \frac{1}{q}
$$

SC capacity is expressed as:

$$
\begin{aligned}
C_{\mathrm{SC}} & =\log _{2}\left(1+\gamma \cdot \max _{q}\left|h_{q}\right|^{2}\right) \\
& =\max _{q}\left\{\log _{2}\left(1+\gamma \cdot\left|h_{q}\right|^{2}\right)\right\} \quad ; 1 \leq q \leq N_{R} \quad \text { bits } / \mathrm{s} / \mathrm{Hz}
\end{aligned}
$$

The ergodic capacity curves for all three combining strategies are shown in Fig. 18. MRC yields best performances in terms of channel capacity. However, MRC is the optimal combining technique, MRC is seldom implemented in a multipath fading channel since the complexity of the receiver is directly resolvable paths (Zhou \& Okamoto, 2004). In general, EGC performs worse than does MRC. Obviously, lower capacity is obtained with SC since only one Radio Frequency (RF) channel is selected at the receiver. A study of combining techniques in terms of BER was presented in (Zhou \& Okamoto, 2004). MRC steel achieves the best BER performances.

\section{Beamforming processing in MIMO systems}

Beamforming is the process of trying to steer the digital baseband signals to one particular direction by weighting these signals differently. This is named "digital beamforming" and we call it beamforming for the sake of brevity, (Jafarkhani, 2005). The desired signal is then obtained by summing the weighted baseband signals. 


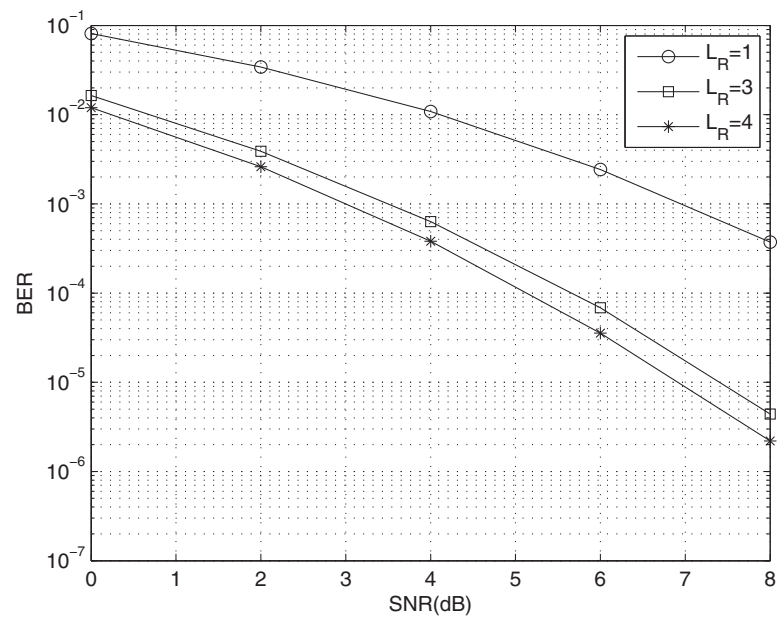

Fig. 18. Capacity for $\mathrm{MIMO}(4 \times 1)$ using various combining techniques-Rayleigh fading channel

\subsection{Beamforming based on SVD decomposition}

In this section, we provide an overview of MIMO systems that use beamforming at both the ends of the communication link. We consider a MIMO system with $N_{T}$ transmit and $N_{R}$ receive dimensions. From a mathematical point of view, joint Transmit-Receive beamforming is based on the minimization (or maximization) of some cost function such as SNR maximization. This method includes determining the transmit beamforming coefficients and the receive beamforming coefficients so that to steer relatively all transmit energy and receive energy in the directions of interest. Joint Transmit-Receive beamforming is illustrated in Fig. 19.
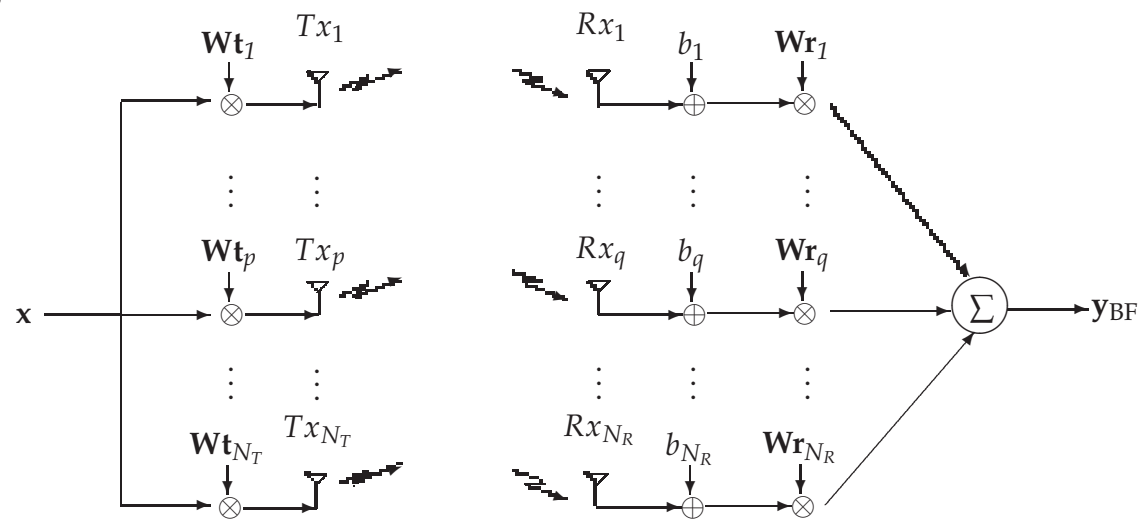

Fig. 19. Joint Transmit-Receive beamforming

- $\mathbf{x}$ : The transmit signal

- $\mathbf{W t}=\left[\mathbf{W t}_{1}, \ldots, \mathbf{W t}_{N_{T}}\right]^{T}$ : The $\left(N_{T} \times 1\right)$ Transmit beamforming vector 
- $\mathbf{H}$ : The $\left(N_{R} \times N_{T}\right)$ channel matrix

- $\mathbf{W r}=\left[\mathbf{W r}_{1}, \ldots, \mathbf{W r}_{N_{R}}\right]^{T}$ : The $\left(N_{R} \times 1\right)$ Receive beamforming vector

- $\mathbf{b}=\left[b_{1}, \ldots, b_{N_{R}}\right]^{T}$ : The $\left(N_{R} \times 1\right)$ Additive noise vector with variance $\sigma_{b}^{2}$

- $\mathbf{y}_{\mathrm{BF}}$ : The output signal

Joint Transmit-Receive beamforming can be described by equation (70).

$$
\mathbf{y}_{\mathrm{BF}}=\mathbf{W r}^{\mathrm{H}} \mathbf{H} \mathbf{W}_{\mathbf{t}} \cdot \mathbf{x}+\mathbf{W r}^{\mathrm{H}} \cdot \mathbf{b}
$$

Eigen-beamforming could be performed by using eigenvectors to find the linear beamformer that optimizes the system performances. Thus, we exploit the SVD factorization for channel matrix $\mathbf{H}\left(\mathbf{H}=\mathbf{U S V}^{H}\right)$. Assigning $\mathbf{U}$ and $\mathbf{V}$ respectively to $\mathbf{W r}$ and $\mathbf{W t}$ is optimal for maximizing the SNR given by :

$$
\mathrm{SNR}_{\mathrm{BF}}=\frac{\left\|\mathbf{W} \mathbf{r}^{H} \mathbf{H W} \mathbf{t}\right\|^{2} \mathrm{E}\left(\mathbf{x} \mathbf{x}^{H}\right)}{\sigma_{b}^{2}\|\mathbf{W r}\|^{2}}
$$

When SVD factorization is applied to MIMO channel matrix, equation (70) becomes :

$$
\mathbf{y}_{\mathrm{BF}}=\mathbf{S} \cdot \mathbf{x}+\mathbf{U}^{H} \cdot \mathbf{b}
$$

Note that Beamforming (Ibnkahla, 2009) is considered as a form of linear combining techniques which are intended to maximize the spectral efficiency. The received SNR for communication system with beamforming is expressed as :

$$
\gamma_{\mathrm{BF}}=\gamma_{r} \cdot \lambda_{\max }(\mathbf{H})
$$

$\lambda_{\max }$ is the maximum eigenvalue associated to matrix $\mathbf{S}$ and $\gamma_{r}$ is the mean received SNR. Thereafter, the capacity for MIMO system with beamforming is expressed as :

$$
C_{\mathrm{BF}}=\log _{2}\left\{1+\gamma_{r} \cdot \lambda_{\max }(\mathbf{H})\right\} \quad \text { bits } / \mathrm{s} / \mathrm{Hz}
$$

Simulation results for MIMO capacity where beamforming technique is performed are shown in Fig. 20. The MIMO channel capacity with beamforming is improved thanks to the spatial diversity.

Note that beamforming technique is shown to improve the performance of the communication link in terms of BER. Fig. 21 shows the plotted curves of BER as a function of SNR relative to three cases :

- System performing beamforming

- Transmission without applying beamforming

- Transmission with simply Zero Forcing (ZF) equalization

The MIMO $(3 \times 3)$ channel is randomly generated and input signal is BPSK modulated. We adopt the correlated MIMO channel with a spreading angle of $90^{\circ}$ and an antenna spacing of $\frac{\lambda}{2}$. Fig. 21 shows that associated SVD beamforming technique brings the best performances in terms of BER. 


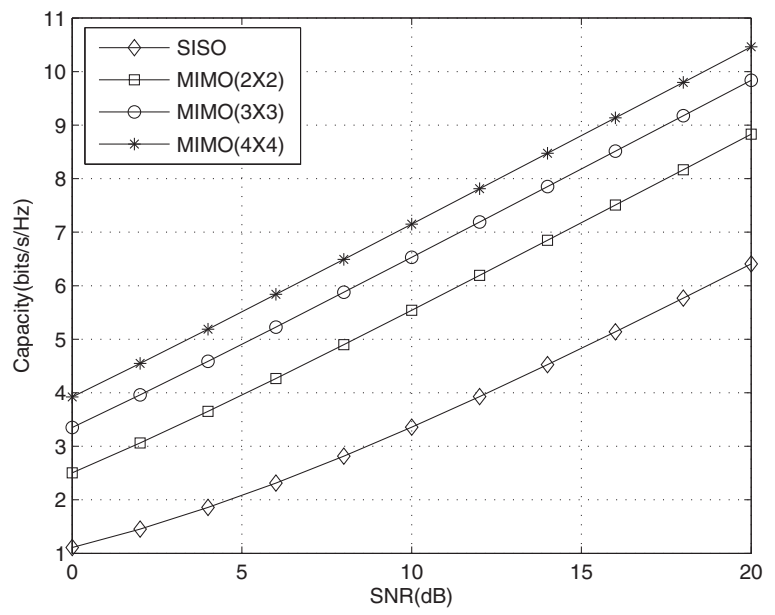

Fig. 20. Capacity of MIMO system with beamforming technique

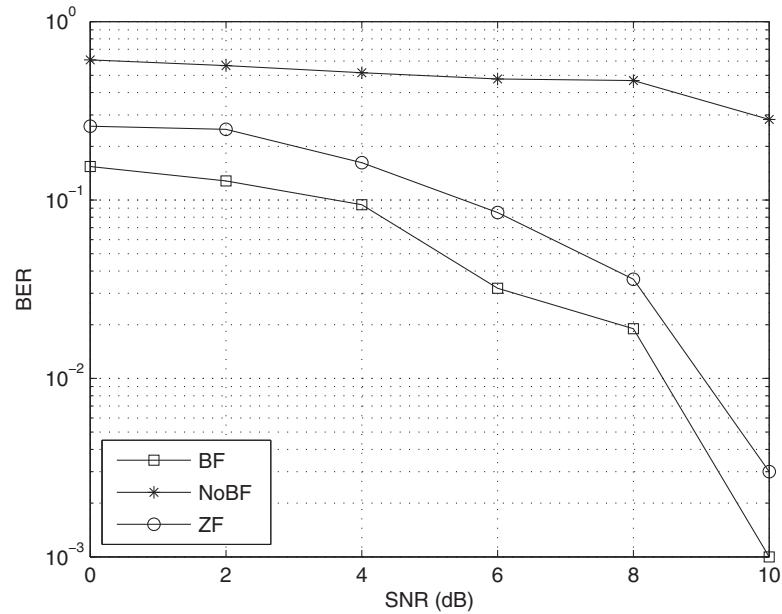

Fig. 21. SVD based beamforming technique 


\subsection{SINR maximization beamforming}

Interference often occurs in wireless propagation environment. When several terminals are densely deployed in the coverage area, Signal to Interference Noise Ratio (SINR) grows up and efficient techniques are required to be implemented. Beamforming is an efficient strategy that could be exploited so that to mitigate interference. Maximizing the SINR criteria could be also considered so that to obtain optimal beamforming weights.

\section{SINR maximization based beamforming in Multi user system Model description}

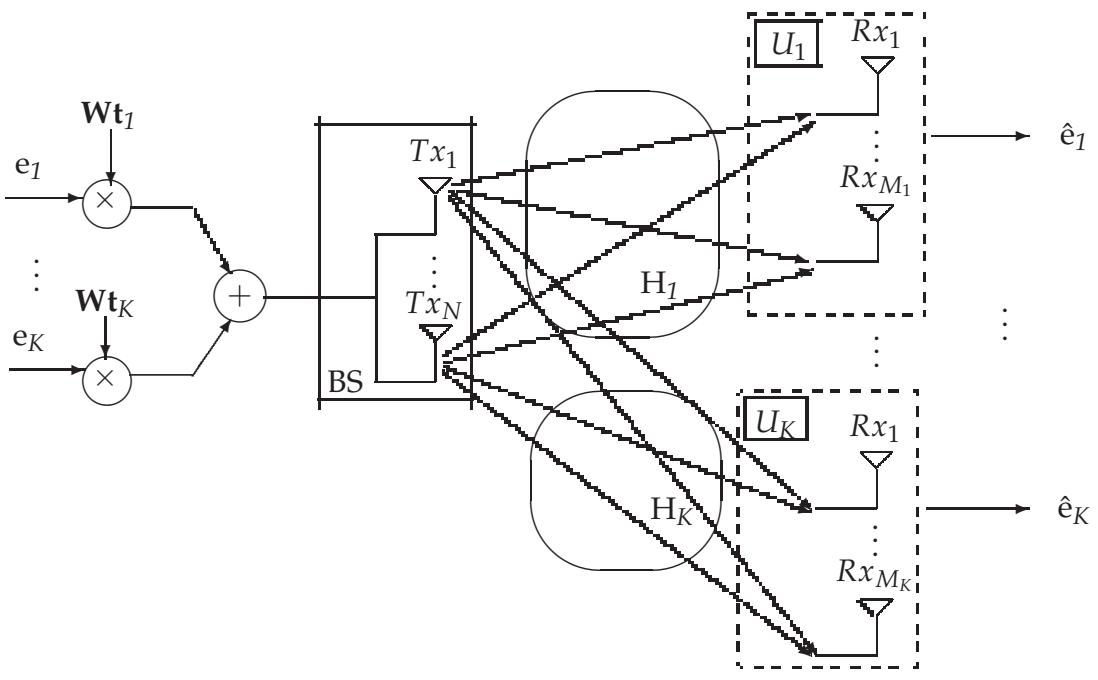

Fig. 22. Multi user system with beamforming

We denote :

- $K$ : Number of users.

- $\mathbf{E}=\left[\mathrm{e}_{1}, \ldots, \mathrm{e}_{N}\right]^{T}$ : The transmit signal vector

- $\mathbf{W t}=\left[\mathbf{W t}_{1}, \ldots, \mathbf{W t}_{K}\right]^{T}$ : Weight vector for beamforming

- $M_{1}, \ldots, M_{K}$ number of antennas respectively for users $U_{1}, \ldots, U_{K}$

- $\mathbf{x}$ : The transmit vector signal of size $(N \times 1)$

Transmit signal is expressed as :

$$
\mathbf{x}=\sum_{k=1}^{K} \mathbf{W} \mathbf{t}_{k} \cdot \mathbf{e}_{k}
$$

We assume that transmit signals and beamforming weights are normalized. The received signal (Of size $\left.\left(M_{i} \times 1\right)\right)$ by user $U_{i}$ is :

$$
\mathrm{y}_{i}=\mathrm{H}_{i} \sum_{k=1}^{K} \mathbf{W t}_{k} \cdot \mathrm{e}_{k}+\mathrm{b}_{i}
$$


$\mathrm{b}_{i}$ is the additive noise with variance $\sigma_{i}^{2}$. The channel matrix $\mathrm{H}_{i}\left(M_{i} \times N\right)$ between user $U_{i}$ with $M_{i}$ antennas and the $N$ antennas at the Base Station (BS) is assumed to be normalized. User $U_{i} ; i=1, \ldots, K$ receives the signal :

$$
\mathrm{y}_{i}=\mathrm{H}_{i} \mathbf{W} \mathbf{t}_{i} \cdot \mathrm{e}_{i}+\sum_{k=1, k \neq i}^{K} \mathrm{H}_{i} \mathbf{W} \mathbf{t}_{k} \cdot \mathrm{e}_{k}+\mathrm{b}_{i}
$$

At the receiver, the estimated signal for user $i$ is:

$$
\hat{\mathrm{e}}_{i}=\frac{\mathbf{W t}_{i}^{H} \mathrm{H}_{i}^{H} \mathrm{y}_{i}}{\left\|\mathrm{H}_{i} \mathbf{W t}_{i}\right\|}
$$

The SINR is the ratio of the received strength of the desired signal to the received strength of undesired signals (Noise + Interference). Associated SINR to user $i$ is expressed as :

$$
\operatorname{SINR}_{i}=\frac{\left\|\mathrm{H}_{i} \mathbf{W} \mathbf{t}_{i}\right\|^{2}}{\left(\sum_{k=1, k \neq i}^{K}\left\|\mathrm{H}_{i} \mathbf{W} \mathbf{t}_{k}\right\|^{2}\right)+\sigma_{i}^{2}}
$$

SINR could also be written as :

$$
\operatorname{SINR}_{i}=\frac{\left\|\mathrm{H}_{i} \mathbf{W t}_{i}\right\|^{2}}{\left(\frac{\sum_{k=1, k \neq i}^{K}\left\|\mathbf{W t}_{i}^{H} \mathrm{H}_{i}^{H} \mathrm{H}_{i} \mathbf{W} \mathbf{t}_{k}\right\|^{2}}{\left\|\mathrm{H}_{i} \mathbf{W t}_{i}\right\|^{2}}\right)+\sigma_{i}^{2}}
$$

Optimal beamformer weights are obtained by maximizing the Signal Leakage Ratio (SLR) metric expressed as :

$$
S L R=\frac{\left\|\mathrm{H}_{i} \mathbf{W} \mathbf{t}_{i}\right\|^{2}}{\left\|\tilde{\mathrm{H}}_{i} \mathbf{W} \mathbf{t}_{i}\right\|^{2}}
$$

where :

$$
\tilde{\mathrm{H}}_{i}=\left[\mathrm{H}_{1}^{H}, \ldots, \mathrm{H}_{i-1}^{H}, \mathrm{H}_{i+1}^{H}, \ldots, \mathrm{H}_{\mathrm{K}}^{H}\right]^{H}
$$

The optimal weights $\mathbf{W t}_{i} ; i=1, \ldots, K$ are derived (Tarighat et al., 2005) as the maximum eigenvector of:

$$
\left(\left(\tilde{\mathrm{H}}_{i}{ }^{H} \tilde{\mathrm{H}}_{i}\right)^{-1}\left(\mathrm{H}_{i}^{H} \mathrm{H}_{i}\right)\right)
$$

Simulation results are shown in Fig. 23. These results show that the method is optimal for determining the beamforming weights. Note that better performances in terms of BER are achieved if more transmit antennas are used. 


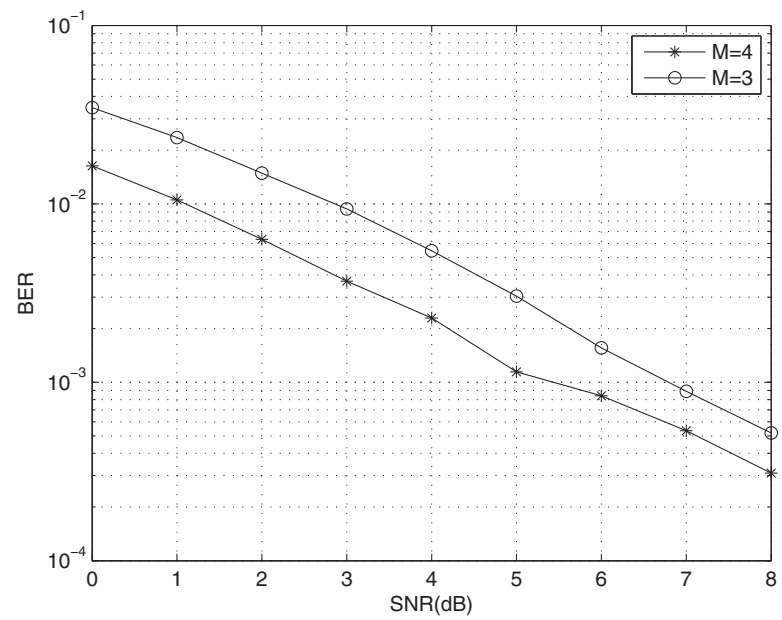

Fig. 23. Multi user $B F(K=3, M=3 / M=4)$

\section{Processing techniques for MIMO systems: Antenna selection}

MIMO system gives high performances in terms of system capacity and reliability of radio communication. Combining techniques such as MRC results in more robust system. Nevertheless, the deployment of multiple antennas would require the implementation of multiple RF chains (Dong et al., 2008). This would be costly in terms of size, power and hardware. For example, when several antennas are deployed, multiple RF chains with separate modulator and demodulator have to be implemented. To overcome these limitations, antenna selection techniques can be applied.

\subsection{Antenna selection}

Antenna selection technique (Ben ZID et al., 2011) is depicted in Fig. 24. We consider a MIMO system with $N_{T}$ transmit antennas and $N_{R}$ receive antennas. The idea of antenna selection is to select $L_{T}$ antennas among the $N_{T}$ transmit antennas and / or $L_{R}$ antennas among the $N_{R}$ receive antennas. We distinguish different forms of antenna selection:

1. Transmit antenna selection

2. Receive antenna selection

3. Hybrid antenna selection: that is when antenna selection is carried among both transmit antennas and receive antennas.
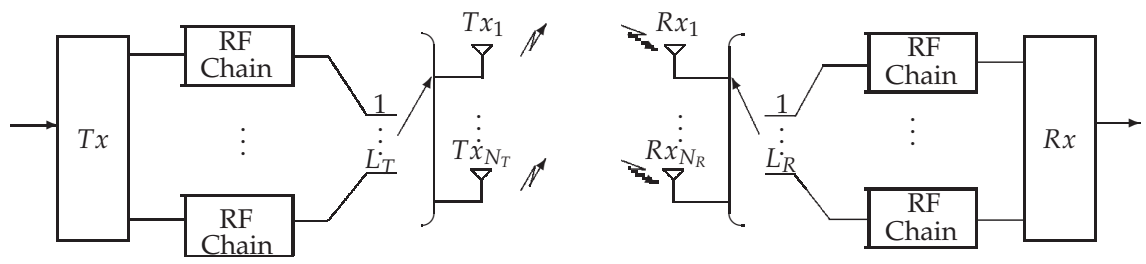

Fig. 24. Antenna selection in MIMO system 
Antenna selection algorithms do not only aim to reduce the system complexity but also to achieve high spectral efficiency. When $L_{T}$ antennas are selected at the transmitter and $L_{R}$ antennas are selected at the receiver, the associated channel will be denoted $\mathbf{H}_{\mathrm{S}}$. The capacity of such system is expressed as :

$$
\begin{aligned}
C_{\text {Sel }} & =\log _{2}\left[\operatorname{det}\left(I_{L_{T}}+\frac{\gamma}{L_{R}} \mathbf{H}_{\mathrm{S}}^{H} \mathbf{H}_{\mathrm{S}}\right)\right] \\
& =\log _{2}\left[\operatorname{det}\left(I_{L_{R}}+\frac{\gamma}{L_{T}} \mathbf{H}_{\mathrm{S}} \mathbf{H}_{\mathrm{S}}^{H}\right)\right]
\end{aligned}
$$

$\gamma$ denotes the SNR. The antenna selection algorithm is intended to find the optimal subset of the transmit antennas and /or the optimal subset of the receive antennas that satisfy capacity system maximization. Nevertheless, it is obvious that the joint antenna selection at the transmitter and the receiver brings more complexity when the number of antennas increases.

\section{Numerical results}

Ergodic capacity of MIMO system with antenna selection at the transmitter and the receiver is shown in Fig. 25. For simulation purposes, we generate a Rayleigh MIMO channel with AWGN. Here, SVD factorization is applied. Plotted curves depict the ergodic capacity for the $\mathrm{MIMO}(4 \times 4)$. This evidently leads to the highest system capacity. When 3 transmit antennas are selected among 4 transmit antennas and 3 receive antennas are selected among 4 receive antennas, the maximum ergodic capacity that could be achieved is plotted in function of SNR. Simulation results are also presented in the case when two antennas are selected at both the transmitter and the receiver. According to the plotted curves in Fig. 25, it is obvious that one of the important limitations of the antenna selection strategy is the important losses in capacity at high SNR regime.

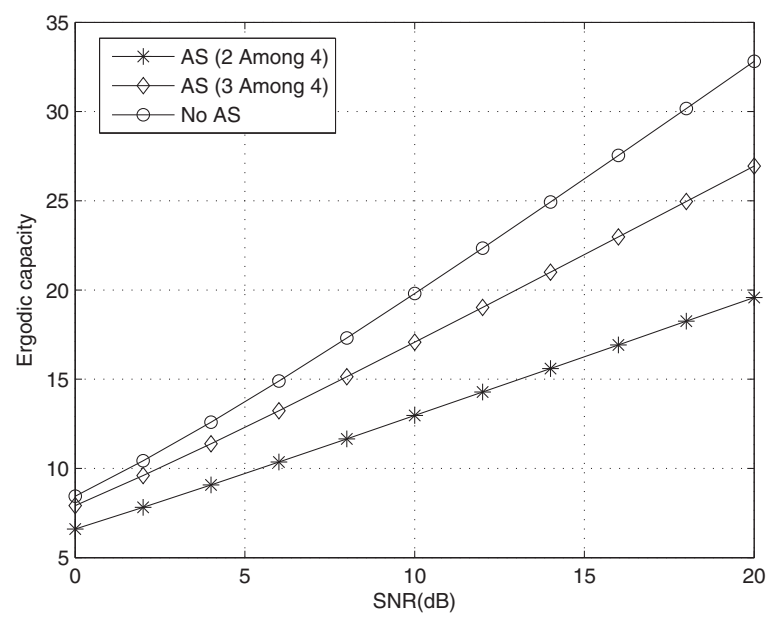

Fig. 25. Antenna selection in MIMO $(4 \times 2)$ : Impact on ergodic capacity 


\subsection{Antenna selection involving ST coding}

We present in this paragraph, the simulation results in terms of average BER when joint Alamouti scheme and antenna selection at the receiver are applied. The MIMO $(4 \times 2)$ system with a Rayleigh channel and AWGN was created. Emitted symbols are QAM (Quadrature Amplitude Modulation) modulated. The simulation model is given by the Fig. $26\left(b_{1}, \ldots, b_{N_{R}}\right.$ denote the additive noise signals). Plotted curves concern subsets of receive antennas where $L_{R}=1$ and $L_{R}=3$. Simulation results show that even with only one selected antenna at the

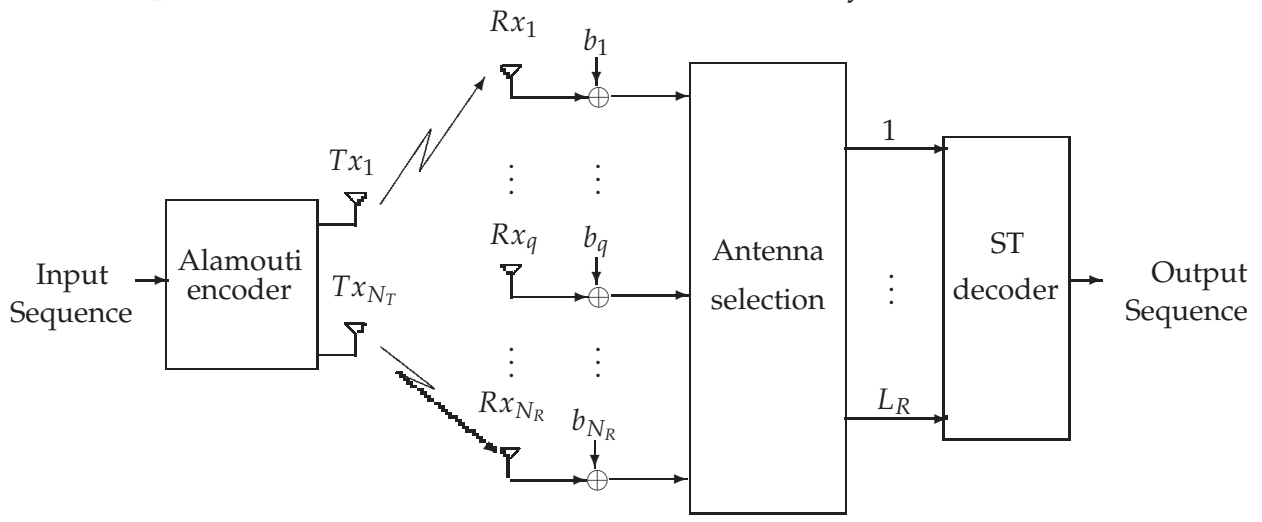

Fig. 26. MIMO system with antenna selection and Alamouti coding

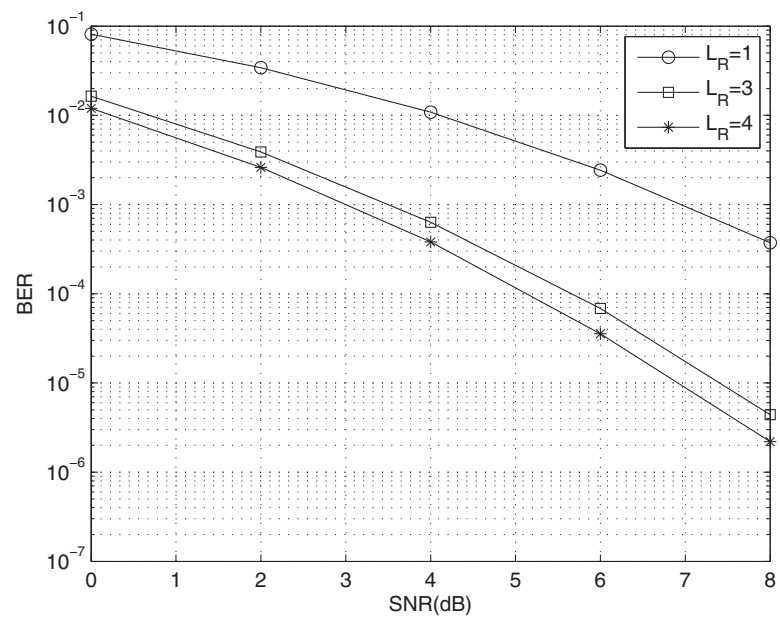

Fig. 27. Joint Alamouti coding and antenna selection in MIMO $(4 \times 2)$

receiver, performances in terms of BER still satisfactory. Nevertheless, when more antennas are selected, better BER values are achieved thanks to receive diversity. 


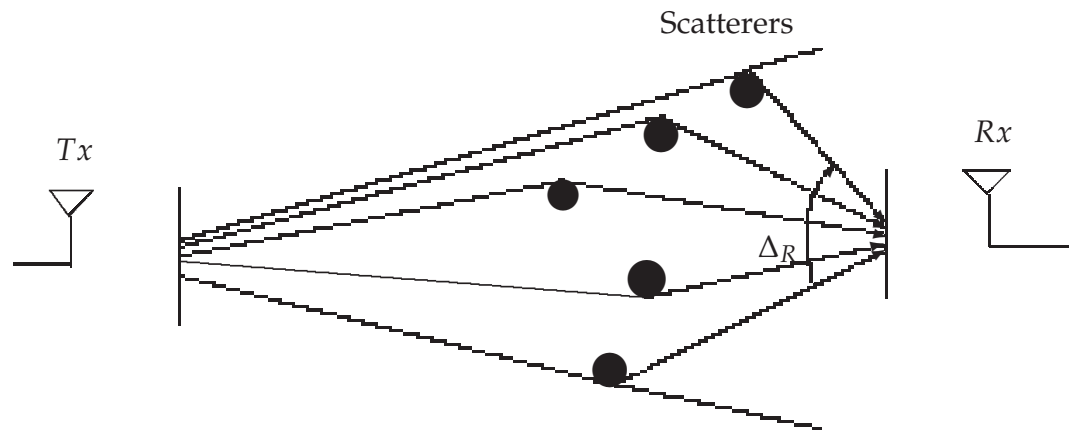

Fig. 28. Angle spread

\subsection{Antenna selection in correlated MIMO channel: Angular dispersion and channel correlation}

Angle spread refers to the spread of DOA of the multipath components at the transmit antenna array. When scatterers are also distributed around the receive antennas, the scattering effect leads also to an angle spreading relative to the DOA. In Fig. 28, the angle spread is denoted $\Delta_{R}$. We present a SISO model rich of local scatterers. For seek of simplicity, we consider a MIMO $\left(N_{R} \times N_{T}\right)$ system with LOS channel and uniform antenna arrays at both the transmitter and the receiver.

We denote :

- $\mathbf{H}$ : MIMO channel matrix

- $d_{q, p}$ : distance between antenna $q$ and antenna $p$

- $\rho_{q, q^{\prime}}:$ correlation coefficient

- $\lambda$ : wavelength

- $\mathbf{R}=\mathrm{E}\left[\mathbf{H}^{H} \mathbf{H}\right]$ Correlation matrix

- $\alpha$ : Angle of arrival

- $p(\alpha)$ : Probability density function of the DOA

- $\Delta_{R}(=2 \pi)$ : Angle spread at the receiving side

When LOS propagation is assumed, the channel coefficients can be expressed as :

$$
h_{q p}=e^{-j 2 \pi \frac{d q, p}{\lambda}} ; q=1, \ldots, N_{R}, p=1, \ldots, N_{T}
$$

The correlation coefficient at the receiving side between two receive antennas of indexes $q$ and $q^{\prime}$ is expressed :

$$
\rho_{q, q^{\prime}}=E\left[\exp \left(-j 2 \pi \frac{d_{q, q^{\prime}} \sin \alpha}{\lambda}\right)\right]
$$

Formula for correlation coefficients is expressed as :

$$
\rho_{q, q^{\prime}}=\int_{\frac{-\Delta_{R}}{2}}^{\frac{\Delta_{R}}{2}} \exp \left(-\frac{j 2 \pi d_{q, q^{\prime}} \sin (\alpha)}{\lambda}\right) p(\alpha) d \alpha \quad ; q \neq q^{\prime}
$$

Evidently :

$$
\rho_{q, q^{\prime}}=1 \quad ; q=q^{\prime}
$$


Following a uniform distribution, correlation coefficients can be expressed as :

$$
\rho_{q, q^{\prime}}=J_{0}\left(2 \pi \frac{d q, q^{\prime}}{\lambda}\right) \quad q=1, \ldots, N_{R} ; \quad q^{\prime}=1, \ldots, N_{R}
$$

$J_{0}($.$) is the zeroth order Bessel function. When antennas are uncorrelated, \rho_{q, q^{\prime}}=0$ if $q \neq q^{\prime}$. Which induces :

$$
J_{0}\left(2 \pi \frac{d_{q, q^{\prime}}}{\lambda}\right) \simeq 0 \Rightarrow 2 \pi \frac{d_{q, q^{\prime}}}{\lambda} \simeq \pi
$$

Thus, in order to mitigate correlation between antennas, antenna spacing between two antennas should be at least equal to $\frac{\lambda}{2}$. Nevertheless, antenna correlation still depends on angular dispersion. Fig. 29 presents the plotted curves of the spatial correlation as a function of the antenna spacing divided by the wavelength for various values of angle spread. According to simulation results depicted in Fig. 29, we conclude that spatial correlation between two antennas depends on antenna spacing and is reduced by higher angle spread.

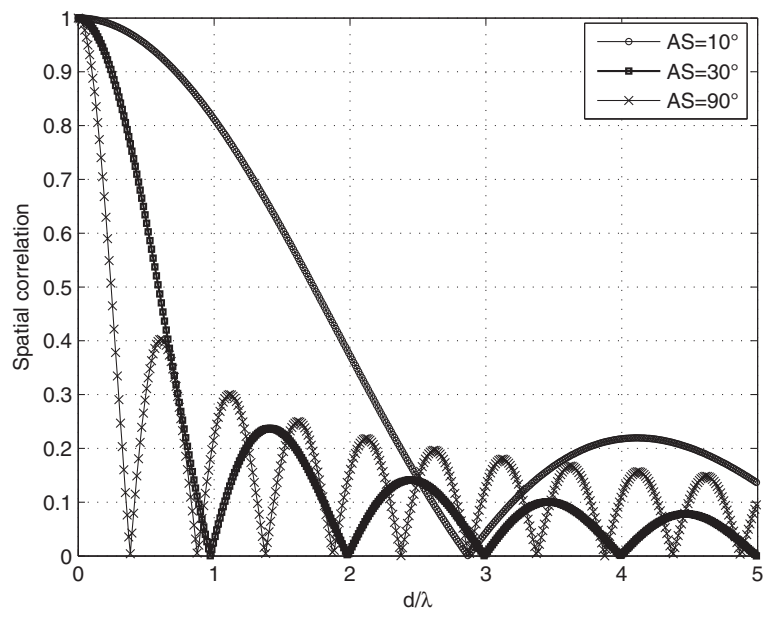

Fig. 29. Impact of angle spread on spatial correlation

Better performances in terms of BER are achieved for $A S=30^{\circ}$. This is due to the fact that for a given antenna spacing, system correlation is higher for lower angular spread. The impact of angle spread on system performances is depicted in Fig. 30.

\section{Multi polarization techniques}

\subsection{Basic antenna theory and concepts}

We present in this paragraph, some basic concepts related to antenna. A rigorous analysis of the antenna theory and the related concepts is available in (Constantine, 2005). Antenna is a transducer for radiating or receiving radio waves. It ideally radiates all the power delivered to it from the transmitter in a desired direction. The far electric field of the electromagnetic wave is written in spherical coordinates as :

$$
E=E_{\theta}(\theta, \phi) \hat{\theta}+E_{\phi}(\theta, \phi) \hat{\phi}
$$




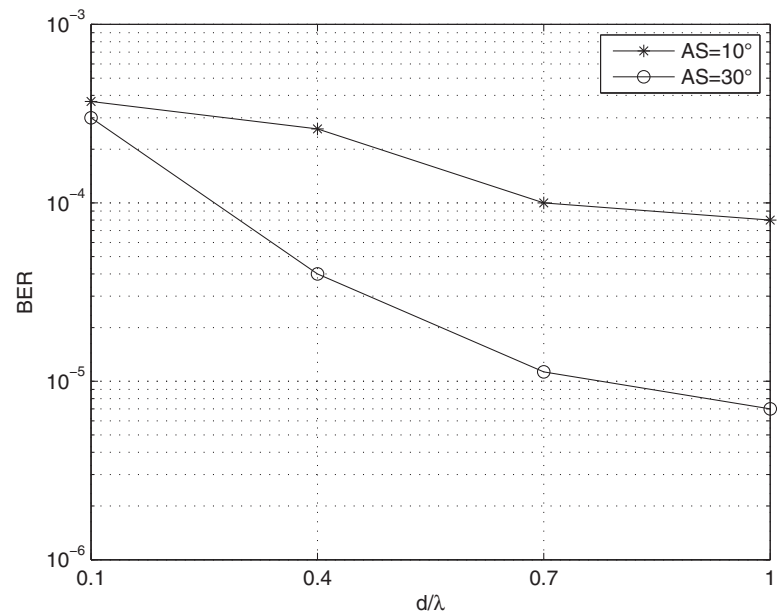

Fig. 30. Impact of angle spread on system performances

$E_{\theta}$ and $E_{\phi}$ are the electric field components. $\theta$ and $\phi$ denote respectively the elevation angle and the azimuthal angle. We distinguish two categories of antennas :

1. Omnidirectional antenna is an antenna system which radiates power uniformly

2. Dipole antenna radiates power in a particular direction.

Electric dipole could be oriented along the x-axis, $y$-axis or the z-axis. Table 2 gives the expressions of the electric field components relative to each antenna orientation.

\begin{tabular}{|l|l|l|}
\hline & $E_{\theta}(\theta, \phi)$ & $E_{\phi}(\theta, \phi)$ \\
\hline $\mathrm{x}$ & $-\cos (\theta) \cos (\phi)$ & $\sin (\phi)$ \\
\hline $\mathrm{y}$ & $-\cos (\theta) \sin (\phi)$ & $-\cos (\phi)$ \\
\hline $\mathrm{z}$ & $\sin (\theta)$ & 0 \\
\hline
\end{tabular}

Table 2. Radiation pattern for electric dipole

\section{Radiation intensity}

Antenna gain is defined as the ratio of the intensity radiated by the antenna divided by the intensity radiated by an isotropic antenna. Normalized radiation intensity (or Antenna gain) is :

$$
\mathbf{G}(\theta, \phi)=\left[\begin{array}{l}
G_{\theta}(\theta, \phi) \\
G_{\phi}(\theta, \phi)
\end{array}\right]=\left[\frac{\sqrt{\frac{1}{4 \pi}\left(\int_{0}^{2 \pi} \int_{0}^{\pi}\left|E_{\theta}(\theta, \phi)\right|^{2} d \Omega+\int_{0}^{2 \pi} \int_{0}^{\pi}\left|E_{\phi}(\theta, \phi)\right|^{2} d \Omega\right)}}{\sqrt{\frac{1}{4 \pi}\left(\int_{\phi}^{2 \pi} \int_{0}^{\pi}\left|E_{\theta}(\theta, \phi)\right|^{2} d \Omega+\int_{0}^{2 \pi} \int_{0}^{\pi}\left|E_{\phi}(\theta, \phi)\right|^{2} d \Omega\right)}}\right]
$$


- $\Omega$ is the beam solid angle through which all the power of the antenna would flow if its radiation intensity is constant for all angles within $\Omega$.

- $G_{\theta}(\theta, \phi)$ and $G_{\phi}(\theta, \phi)$ are respectively the elevation antenna gain and the azimuthal antenna gain.

\subsection{D Geometric wide band channel model}

The 3D Geometric wide band channel model is presented in Fig.31.

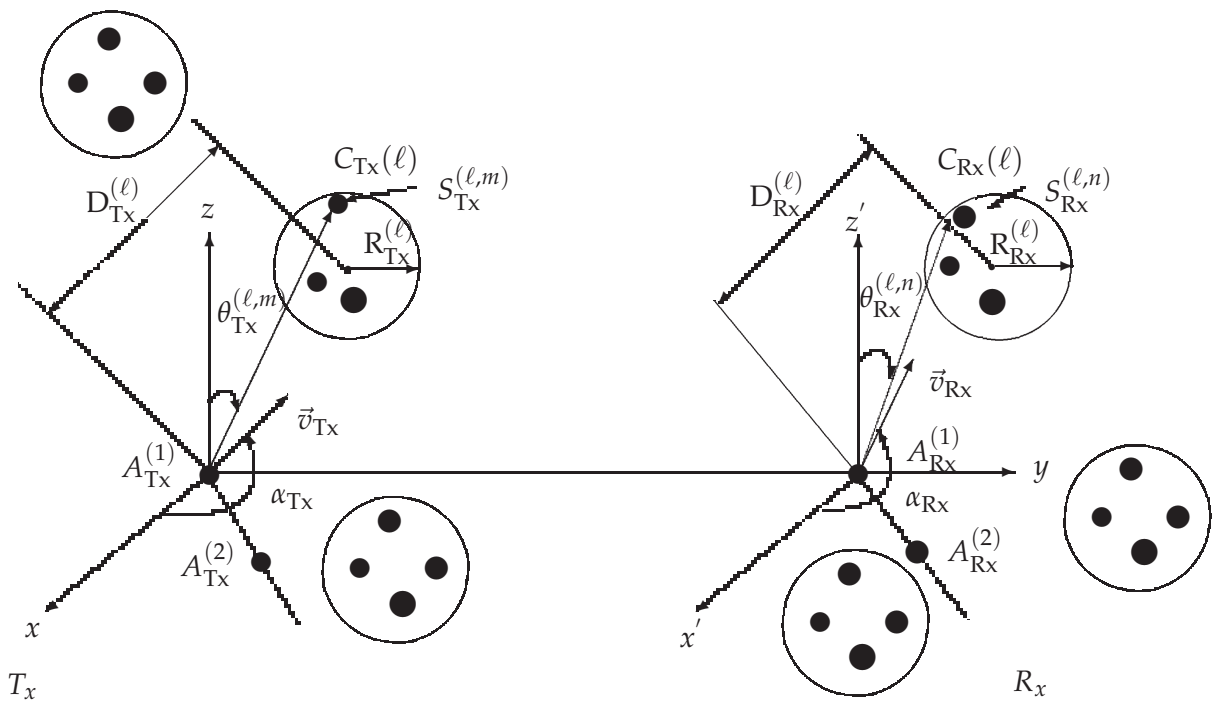

Fig. 31. 3D Geometric model for MIMO channel $\left(N_{R}=2, N_{T}=2\right)$

Two transmit antennas $\left(A_{\mathrm{Tx}}^{(1)}, A_{\mathrm{Tx}}^{(2)}\right)$ and two receive antennas $\left(A_{\mathrm{Rx}}^{(1)}, A_{\mathrm{Rx}}^{(2)}\right)$ are presented. Wide band MIMO channel involves several local clusters of scatterers which are distributed around the transmitter and the receiver. The cluster index is denoted $\ell, \quad \ell=1, \ldots, L$. Cluster around the transmitter $C_{\mathrm{Tx}}(\ell)$ is assumed to be associated with a set of $M^{(\ell)}$ scatterers $\left(S_{\mathrm{Tx}}^{(\ell, m)} ; \quad m=\right.$ $\left.1, \ldots, M^{(\ell)}\right)$. Cluster around the receiver $C_{\mathrm{Rx}}(\ell)$ is assumed to be associated with a set of $N^{(\ell)}$ scatterers $\left(S_{\mathrm{Rx}}^{(\ell, n)} ; \quad n=1, \ldots, N^{(\ell)}\right)$.

We take for notations:

- $\mathrm{R}_{\mathrm{Tx}}^{(\ell)}$ : Transmit cluster radius of index $\ell$

- $\mathrm{D}_{\mathrm{Tx}}^{(\ell)}$ : Distance between the reference transmit antenna and the transmit cluster center

- $d_{1, \ell, m}$ : Distance between antenna $A_{\mathrm{Tx}}^{(1)}$ and a scatterer $S_{\mathrm{Tx}}^{(\ell, m)}$

- $d_{2, \ell, m}$ : Distance between antenna $A_{\mathrm{Tx}}^{(2)}$ and a scatterer $S_{\mathrm{Tx}}^{(\ell, m)}$

- $d_{\mathrm{Tx}}$ : Transmit antennas spacing

- $\mathrm{D}_{\mathrm{Tx} \leftrightarrow \mathrm{Rx}}$ : Distance between the transmitter and the receiver

- $\mathrm{R}_{\mathrm{Rx}}^{(\ell)}$ : Cluster radius at the receiver of index $\ell$ 
- $\mathrm{D}_{\mathrm{Rx}}^{(\ell)}$ : Distance between the reference antenna $A_{\mathrm{Rx}}^{(1)}$ and the center of the cluster $\ell$ at the receiver.

- $d_{1, \ell, n}$ : Distance between antenna $A_{\mathrm{Rx}}^{(1)}$ and the scatterer $S_{\mathrm{Rx}}^{(\ell, n)}$

- $d_{2, \ell, n}$ : Distance between antenna $A_{\mathrm{Rx}}^{(2)}$ and the scatterer $S_{\mathrm{Rx}}^{(\ell, n)}$

The Non-Line of Sight (NLOS) channel coefficients in 3D Geometric model are given by (Prayongpun, 2009):

$$
\begin{aligned}
h_{q p}^{N L o s}(t, f)= & \lim _{M, N \rightarrow \infty} \sum_{\ell=1}^{L} \sqrt{\frac{P D P(\ell)}{M^{(\ell)} N^{(\ell)}}} \sum_{m=1}^{M^{(\ell)}} \sum_{n=1}^{N^{(\ell)}} G_{p}\left(\theta_{\mathrm{Tx}}^{(\ell, m)}, \phi_{\mathrm{Tx}}^{(\ell, m)} ; \beta_{\mathrm{Tx}}, \gamma_{\mathrm{Tx}}\right) a_{\ell m}^{(p)} b_{\ell n}^{(q)} \\
& G_{q}\left(\theta_{\mathrm{Rx}}^{(\ell, n)}, \phi_{\mathrm{Rx}}^{(\ell, n)} ; \beta_{\mathrm{Rx}}, \gamma_{\mathrm{Rx}}\right) \exp \left\{j\left(2 \pi\left(f_{\mathrm{Tx}}^{(\ell, m)}+f_{\mathrm{Rx}}^{(\ell, n)}\right) t+\varphi_{\ell m n}+\varphi_{0}^{(\ell)}\right)\right\}
\end{aligned}
$$

- $q \in\left\{1, \ldots, N_{R}\right\}$

- $p \in\left\{1, \ldots, N_{T}\right\}$

- $P D P(\ell)$ is the power delay profile which gives the intensity of a signal received through a multipath channel connecting a pair of clusters.

- $G_{p}\left(\theta_{\mathrm{Tx}}^{(\ell, m)}, \phi_{\mathrm{Tx}}^{(\ell, m)} ; \beta_{\mathrm{Tx}}, \gamma_{\mathrm{Tx}}\right)$ is the gain of antenna $p$ with associated oriented direction $\left(\beta_{\mathrm{Tx}}, \gamma_{\mathrm{Tx}}\right)$ and a wave propagation direction $\left(\theta_{\mathrm{Tx}}^{(\ell, m)}, \phi_{\mathrm{Tx}}^{(\ell, m)}\right)$.

- $G_{q}\left(\theta_{\mathrm{Rx}}^{(\ell, n)}, \phi_{\mathrm{Rx}}^{(\ell, n)} ; \beta_{\mathrm{Rx}}, \gamma_{\mathrm{Rx}}\right)$ is the gain of antenna $q$ with associated oriented direction $\left(\beta_{\mathrm{Rx}}, \gamma_{\mathrm{Rx}}\right)$ and a wave propagation direction $\left(\theta_{\mathrm{Rx}}^{(\ell, n)}, \phi_{\mathrm{Rx}}^{(\ell, n)}\right)$.

- $a_{\ell m}^{(p)}=\exp \left\{j 2 \pi(p-1)\left(d_{\mathrm{Tx}} / \lambda\right)\left[\cos \left(\theta_{\mathrm{Tx}}^{(\ell, m)}\right) \cos \left(\beta_{\mathrm{Tx}}\right)+\sin \left(\theta_{\mathrm{Tx}}^{(\ell, m)}\right) \sin \left(\beta_{\mathrm{Tx}}\right) \cos \left(\phi_{\mathrm{Tx}}^{(\ell, m)}-\gamma_{\mathrm{Tx}}\right)\right]\right\}$

- $b_{\ell n}^{(q)}=\exp \left\{j 2 \pi(q-1)\left(d_{\mathrm{Rx}} / \lambda\right)\left[\cos \left(\theta_{\mathrm{Rx}}^{(\ell, n)}\right) \cos \left(\beta_{\mathrm{Rx}}\right)+\sin \left(\theta_{\mathrm{Rx}}^{(\ell, n)}\right) \sin \left(\beta_{\mathrm{Rx}}\right) \cos \left(\phi_{\mathrm{Rx}}^{(\ell, n)}-\gamma_{\mathrm{Rx}}\right)\right]\right\}$

- $f_{\mathrm{Tx}}^{(\ell, m)}=\left(\left|\vec{v}_{\mathrm{Tx}}\right| / \lambda\right) \sin \left(\theta_{\mathrm{Tx}}^{(\ell, m)}\right) \cos \left(\phi_{\mathrm{Tx}}^{(\ell, m)}-\alpha_{\mathrm{Tx}}\right)$

- $f_{\mathrm{Rx}}^{(\ell, n)}=\left(\left|\vec{v}_{\mathrm{Rx}}\right| / \lambda\right) \sin \left(\theta_{\mathrm{Rx}}^{(\ell, n)}\right) \cos \left(\phi_{\mathrm{Rx}}^{(\ell, n)}-\alpha_{\mathrm{Rx}}\right)$

- $\varphi_{0}^{(\ell)}=-2 \pi\left(\mathrm{D}_{\mathrm{Tx}}^{(\ell)}+\mathrm{D}_{\mathrm{Tx} \leftrightarrow \mathrm{Rx}}+\mathrm{D}_{\mathrm{Rx}}^{(\ell)}\right) / \lambda$

- $\varphi_{\ell m n} \sim U[-\pi, \pi]$

We assume that the transmitter and the receiver have motions above the plan $(x, y)$ with relative directions $\alpha_{\mathrm{Tx}}$ and $\alpha_{\mathrm{Rx}}$. In 3D Geometric model, the distances are expressed as :

$$
\begin{aligned}
d_{1, \ell, m} & \approx \mathrm{D}_{\mathrm{Tx}}^{(\ell)} \\
d_{2, \ell, m} & \approx \mathrm{D}_{\mathrm{Tx}}^{(\ell)}-d_{\mathrm{Tx}} \cos \left(\theta_{\mathrm{Tx}}^{(\ell, m)}\right) \cos \left(\beta_{\mathrm{Tx}}\right)-d_{\mathrm{Tx}} \sin \left(\theta_{\mathrm{Tx}}^{(\ell, m)}\right) \sin \left(\beta_{\mathrm{Tx}}\right) \cos \left(\phi_{\mathrm{Tx}}^{(\ell, m)}-\gamma_{\mathrm{Tx}}\right) \\
d_{1, \ell, n} & \approx \mathrm{D}_{\mathrm{Rx}}^{(\ell)} \\
d_{2, \ell, n} & \approx \mathrm{D}_{\mathrm{Rx}}^{(\ell)}-d_{\mathrm{Rx}} \cos \left(\theta_{\mathrm{Rx}}^{(\ell, n)}\right) \cos \left(\beta_{\mathrm{Rx}}\right)-d_{\mathrm{Rx}} \sin \left(\theta_{\mathrm{Rx}}^{(\ell, n)}\right) \sin \left(\beta_{\mathrm{Rx}}\right) \cos \left(\phi_{\mathrm{Rx}}^{(\ell, n)}-\gamma_{\mathrm{Rx}}\right) \\
d_{\ell, m, n} & \approx \mathrm{D}_{\mathrm{Tx} \leftrightarrow \mathrm{Rx}}+\mathrm{D}_{\mathrm{Rx}}^{(\ell)} \sin \left(\theta_{\mathrm{Rx}}^{(\ell, n)}\right) \sin \left(\phi_{\mathrm{Rx}}^{(\ell, n)}\right)-\mathrm{D}_{\mathrm{Tx}}^{(\ell)} \sin \left(\theta_{\mathrm{Tx}}^{(\ell, m)}\right) \sin \left(\phi_{\mathrm{Tx}}^{(\ell, m)}\right) \approx \mathrm{D}_{\mathrm{Tx} \leftrightarrow \mathrm{Rx}}
\end{aligned}
$$

When there are no scatterers around the transmitter or the receiver, the channel model is referred as Line of Sight (LOS). 


\subsection{Depolarization phenomena}

The polarization of an antenna is defined as the polarization of the wave radiated by the antenna. At a given position, antenna polarization describes the orientation of the electric field. Suppose an electromagnetic wave radiated by a transmit antenna has an incident electric field $E_{i}$ with two components $E_{i, \theta}$ and $E_{i, \phi}$. In each scatterer's environment, the electric field components are reflected (Fig.32). Reflected elevation and azimuth components of the incident electric field $E_{i}$ are denoted $E_{r, \theta}$ and $E_{r, \phi}$.

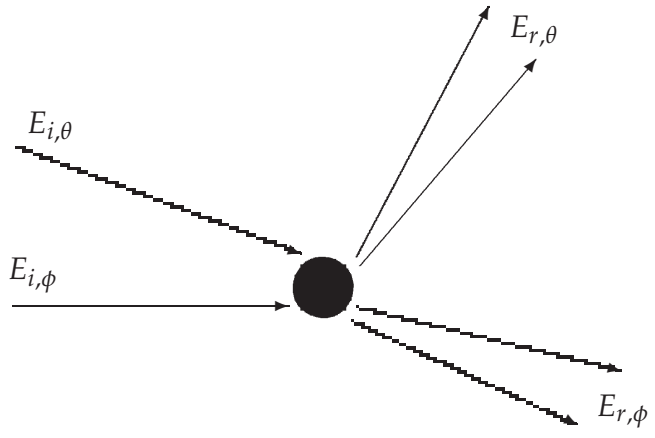

Fig. 32. Antenna depolarization

The radiation pattern is expressed as a function of the azimuth and elevation amplitudes of polarization vectors in both the elevation and the azimuth directions $(\hat{\theta}$ and $\hat{\phi})$.

$$
E_{i}=E_{i, \theta}(\theta, \phi) \hat{\theta}+E_{i, \phi}(\theta, \phi) \hat{\phi}
$$

The reflected electric field is expressed as :

$$
E_{r}=\mathrm{SE}_{i}
$$

where :

- $E_{r}$ is the matrix notation for the reflected electric field expressed as:

$$
E_{r}=\left[\begin{array}{l}
E_{r, \theta} \\
E_{r, \phi}
\end{array}\right]
$$

- $\mathrm{S}$ is the scattering matrix given by:

$$
S=\left[\begin{array}{ll}
v_{\theta \theta} & v_{\theta \phi} \\
v_{\phi \theta} & v_{\phi \phi}
\end{array}\right]
$$

- $v_{\theta \theta}$ and $v_{\phi \phi}$ respectively measure the co-polarization gains relative to the elevation transmission and the azimuth transmission.

- $v_{\theta \phi}$ measures the depolarization gain of the elevation polarization relative to the azimuth polarization.

- $v_{\phi \theta}$ measures the depolarization gain of the azimuth polarization relative to the elevation polarization. 
- The matrix notation for the incident electric field is :

$$
E_{i}=\left[\begin{array}{l}
E_{i, \theta} \\
E_{i, \phi}
\end{array}\right]
$$

The depolarization effect is characterized by the Cross Polarization Discrimination (XPD)(Raoof \& Zhou, 2009)(Raoof \& Prayongpun, 2007) which is defined as the power ratio of the co-polarization and cross-polarization components of the mean incident wave. The polarization discrimination for the elevation transmission is :

$$
X P D_{\theta}=\frac{\mathrm{E}\left|v_{\theta \theta}\right|^{2}}{\mathrm{E}\left|v_{\phi \theta}\right|^{2}}
$$

The polarization discrimination for the azimuth transmission is :

$$
X P D_{\phi}=\frac{\mathrm{E}\left|v_{\phi \phi}\right|^{2}}{\mathrm{E}\left|v_{\theta \phi}\right|^{2}}
$$

In the following, we denote :

$$
\chi_{\theta}=\frac{1}{X P D_{\theta}} \quad \text { and } \quad \chi_{\phi}=\frac{1}{X P D_{\phi}}
$$

\section{GSCM channel model involving antenna depolarization}

The wide band GSCM channel modeling with antenna depolarization is given by :

$$
\begin{aligned}
h_{q p}^{N L O S}(t, f)= & \lim _{M, N \rightarrow \infty} \sum_{\ell=1}^{L} \sqrt{\frac{P D P(\ell)}{M^{(\ell)} N^{(\ell)}}} \sum_{m=1}^{M^{(\ell)}} \sum_{n=1}^{N^{(\ell)}} a_{m}^{(p)} b_{n}^{(q)} \exp \left\{j\left(2 \pi\left(f_{\mathrm{Tx}}^{(\ell, m)}+f_{\mathrm{Rx}}^{(\ell, n)}\right) t+\varphi_{\ell m n}+\varphi_{0}^{\ell}\right)\right\} \\
& {\left[\begin{array}{l}
G_{q, \theta}\left(\theta_{\mathrm{Rx}}^{(\ell, n)}, \phi_{\mathrm{Rx}}^{(\ell, n)} ; \beta_{\mathrm{Rx}}, \gamma_{\mathrm{Rx}}\right) \\
G_{q, \phi}\left(\theta_{\mathrm{Rx}}^{(\ell, n)}, \phi_{\mathrm{Rx}}^{(\ell, n)} ; \beta_{\mathrm{Rx}}, \gamma_{\mathrm{Rx}}\right)
\end{array}\right]^{T} \mathrm{~S}_{\mathrm{Tx}, \mathrm{Rx}}^{(\ell, m, n)}\left[\begin{array}{c}
G_{p, \theta}\left(\theta_{\mathrm{Tx}}^{(\ell, m)}, \phi_{\mathrm{Tx}}^{(\ell, m)} ; \beta_{\mathrm{Tx}}, \gamma_{\mathrm{Tx}}\right) \\
G_{p, \phi}\left(\theta_{\mathrm{Tx}}^{(\ell, m)}, \phi_{\mathrm{Tx}}^{(\ell, m)} ; \beta_{\mathrm{Tx}}, \gamma_{\mathrm{Tx}}\right)
\end{array}\right] \quad(102) }
\end{aligned}
$$

The scattering matrix with the depolarization mechanism is expressed as :

$$
\mathrm{S}_{\mathrm{Tx}, \mathrm{Rx}}^{(\ell, m, n)}=\left[\begin{array}{l}
\sqrt{\frac{1}{1+\chi_{\theta}^{(\ell, m, n)}}} \exp \left(j \varepsilon_{\theta \theta}^{(\ell, m, n)}\right) \sqrt{\frac{\chi_{\phi}^{(\ell, m, n)}}{1+\chi_{\phi}^{(\ell, m, n)}}} \exp \left(j \varepsilon_{\theta \phi}^{(\ell, m, n)}\right) \\
\sqrt{\frac{\chi_{\theta}^{(\ell, m, n)}}{1+\chi_{\theta}^{(\ell, m, n)}}} \exp \left(j \varepsilon_{\phi \theta}^{(\ell, m, n)}\right) \sqrt{\frac{1}{1+\chi_{\phi}^{(\ell, m, n)}}} \exp \left(j \varepsilon_{\phi \phi}^{(\ell, m, n)}\right)
\end{array}\right]
$$

where $\varepsilon_{\theta \theta}^{(\ell, m, n)}, \varepsilon_{\theta \phi}^{(\ell, m, n)}, \varepsilon_{\phi \theta}^{(\ell, m, n)}$ and $\varepsilon_{\phi \phi}^{(\ell, m, n)}$ denote the phase offsets.

\subsection{Impact of XPD on system capacity}

We examine the impact of the XPD parameter on MIMO system capacity. For the seek of simplicity, we consider the $(2 \times 2)$ MIMO channel generated according to the Kronecker channel modeling. The dual polarized MIMO system is adopted (Fig. 33). Assuming that the CSI is known at the receiver side, the MIMO system capacity can be derived by exploiting the SVD technique. Fig.34 shows plotted curves of the CCDF for various inverse XPDs and the curve of the MIMO channel capacity as a function of the XPD. Simulation results show that 


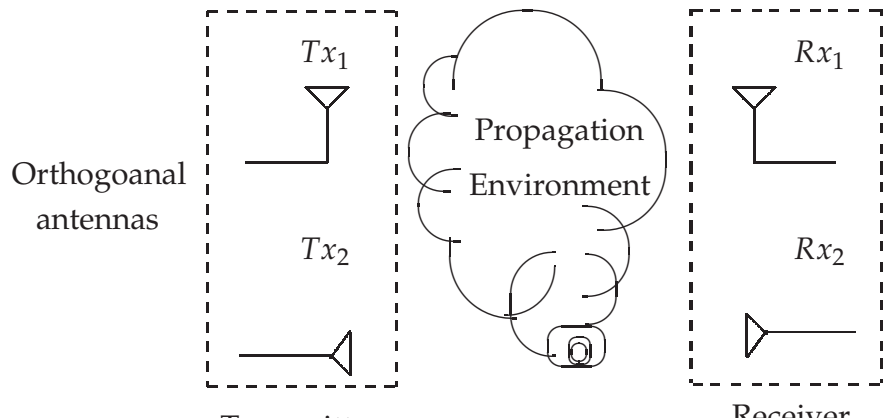

Transmitter

Receiver

Fig. 33. Dual polarized antennas

XPD affects the performances of the MIMO system in terms of the CCDF and the capacity. The MIMO system capacity is shown to be seriously reduced for high level of the polarization discrimination. Thus, mismatch in polarization results in losses in the MIMO channel capacity.

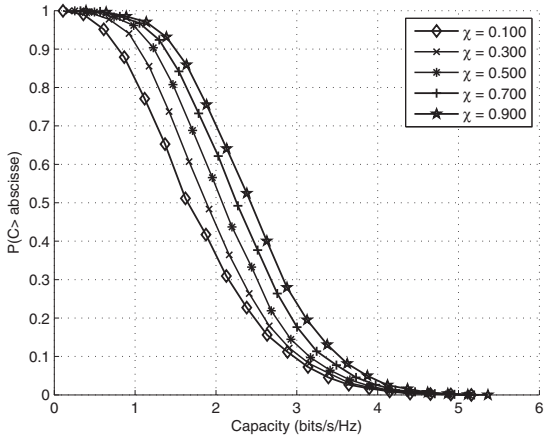

(a)

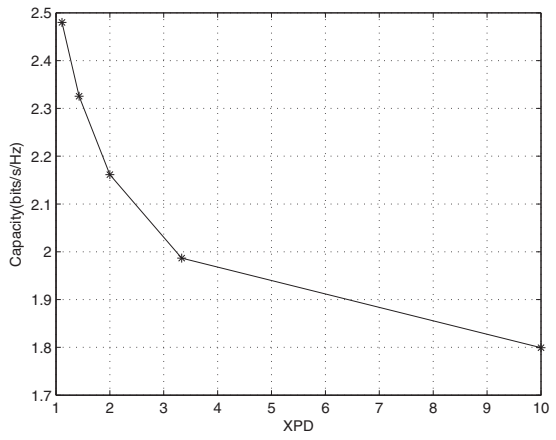

(b)

Fig. 34. Impact of XPD:(a) CCDF for dual polarized MIMO system, (b) Dual polarized MIMO system capacity

\subsection{GSCM channel correlation}

Correlation coefficients relative to the NLOS channels $h_{q p}^{N L O S}(t, \tau)$ and $h_{\tilde{q} \tilde{p}}^{N L O S}(t, \tau)$ are expressed as :

$$
R_{q p, \tilde{q} \tilde{p}}\left(\xi, \tau-\tau^{\prime}\right)=E\left[h_{q p}(t, \tau) h_{\tilde{q} \tilde{p}}^{*}\left(t-\xi, \tau^{\prime}\right)\right]
$$

For zero phase offsets and one cluster of scatterers $(L=1)$, if $\chi_{\theta}=\chi_{\phi}=\chi$, then equation (102) reduces to :

$$
\begin{aligned}
R_{q p, \tilde{q} \tilde{p}}(\xi)= & \lim _{M, N \rightarrow \infty} \frac{1}{M N} \sum_{m=1}^{M} \sum_{n=1}^{N} a_{m}^{(p)} b_{n}^{(q)} a_{m}^{(\tilde{p})^{*}} b_{n}^{(\tilde{q})^{*}} \exp \left\{j\left(2 \pi\left(f_{\mathrm{Tx}}^{(m)}+f_{\mathrm{Rx}}^{(n)}\right)\right) \xi\right\} \\
& {\left[\begin{array}{ll}
G_{q, \theta} \cdot G_{\tilde{q}, \theta} \\
G_{q, \phi} \cdot G_{\tilde{q}, \phi}
\end{array}\right]^{T}\left[\begin{array}{cc}
1 & \chi \\
\chi & 1
\end{array}\right]\left[\begin{array}{c}
G_{p, \theta} \cdot G_{\tilde{p}, \theta} \\
G_{p, \phi} \cdot G_{\tilde{p}, \phi}
\end{array}\right] }
\end{aligned}
$$


The continuous spatial correlation coefficients are given by (Prayongpun, 2009):

$$
\begin{aligned}
& R_{q p, \tilde{q} \tilde{p}}(\xi)=\iiint \int \exp \left\{j \frac{2 \pi(\tilde{p}-p) d_{\mathrm{Tx}}}{\lambda} \sin \theta_{\mathrm{Tx}} \cos \phi_{\mathrm{Tx}}-j 2 \pi f_{\mathrm{Tx}} \xi \sin \theta_{\mathrm{Tx}} \cos \left(\phi_{\mathrm{Tx}}-\gamma_{\mathrm{Tx}}\right)\right\} \\
& \exp \left\{j \frac{2 \pi(\tilde{q}-q) d_{\mathrm{Rx}}}{\lambda} \sin \theta_{\mathrm{Rx}} \cos \phi_{\mathrm{Rx}}-j 2 \pi f_{\mathrm{Rx}} \xi \sin \theta_{\mathrm{Rx}} \cos \left(\phi_{\mathrm{Rx}}-\gamma_{\mathrm{Rx}}\right)\right\} \\
& {\left[\begin{array}{c}
G_{q, \theta} \cdot G_{\tilde{q}, \theta} \\
G_{q, \phi} \cdot G_{\tilde{q}, \phi}
\end{array}\right]^{T}\left[\begin{array}{cc}
1 & \chi \\
\chi & 1
\end{array}\right]\left[\begin{array}{c}
G_{p, \theta} \cdot G_{\tilde{p}, \theta} \\
G_{p, \phi} \cdot G_{\tilde{p}, \phi}
\end{array}\right] p\left(\gamma_{\mathrm{Tx}}\right) p\left(\gamma_{\mathrm{Rx}}\right) p\left(\Omega_{\mathrm{Tx}}\right) p\left(\Omega_{\mathrm{Rx}}\right) d \gamma_{\mathrm{Tx}} d \gamma_{\mathrm{Rx}} d \Omega_{\mathrm{Tx}} d \Omega_{\mathrm{Rx}}}
\end{aligned}
$$

- $\Omega$ involves the elevation angle and the azimuth angle and $d \Omega=\sin \theta d \phi d \theta$.

- $p\left(\Omega_{\mathrm{Tx}}\right)\left(=p\left(\theta_{\mathrm{Tx}}, \phi_{\mathrm{Tx}}\right)\right)$ and $p\left(\Omega_{\mathrm{Rx}}\right)\left(=p\left(\theta_{\mathrm{Rx}}, \phi_{\mathrm{Rx}}\right)\right)$ respectively denote the probability distribution of the scatterers around the transmitter and the probability distribution of scatterers around the receiver.

The spatial correlation coefficients $R_{q p, \tilde{q} \tilde{p}}(\tilde{\xi})$ is obtained as the product of three terms:

1. Transmit spatial correlation: $R \cdot p, \tilde{p}(\xi)$

2. Receive spatial correlation: $R_{q \cdot, \tilde{q} \cdot}(\xi)$

3. Polarization correlation: $\mho$

where :

$$
\begin{aligned}
R \cdot p, \tilde{p}(\xi)= & \iint\left[\begin{array}{c}
G_{p, \theta} \cdot G_{\tilde{p}, \theta} \\
G_{p, \phi} \cdot G_{\tilde{p}, \phi}
\end{array}\right] \exp \left\{j 2 \pi(\tilde{p}-p) d_{\mathrm{Tx}} \sin \theta_{\mathrm{Tx}} \cos \phi_{\mathrm{Tx}} / \lambda\right\} \cdot \\
& \exp \left\{-j 2 \pi f_{\mathrm{Tx}} \xi \sin \theta_{\mathrm{Tx}} \cos \left(\phi_{\mathrm{Tx}}-\gamma_{\mathrm{Tx}}\right)\right\} p\left(\gamma_{\mathrm{Tx}}\right) p\left(\Omega_{\mathrm{Tx}}\right) d \gamma_{\mathrm{Tx}} d \Omega_{\mathrm{Tx}} \\
R_{q, \tilde{q}} \cdot(\xi)= & \iint\left[\begin{array}{c}
G_{q, \theta} \cdot G_{\tilde{q}, \theta} \\
G_{q, \phi} \cdot G_{\tilde{q}, \phi}
\end{array}\right] \exp \left\{j 2 \pi(\tilde{q}-q) d_{\mathrm{Rx}} \sin \theta_{\mathrm{Rx}} \cos \phi_{\mathrm{Rx}} / \lambda\right\} . \\
& \exp \left\{-j 2 \pi f_{\mathrm{Rx}} \xi \sin \theta_{\mathrm{Rx}} \cos \left(\phi_{\mathrm{Rx}}-\gamma_{\mathrm{Rx}}\right)\right\} p\left(\gamma_{\mathrm{Rx}}\right) p\left(\Omega_{\mathrm{Rx}}\right) d \gamma_{\mathrm{Rx}} d \Omega_{\mathrm{Rx}} \\
\mho= & {\left[\begin{array}{cc}
1 & \chi \\
\chi & 1
\end{array}\right] }
\end{aligned}
$$

In literature, azimuth angles may follow uniform, gaussian, von Mises or Laplacien distributions. For the elevation angle, three distribution functions can be used which are uniform, cosinus or gaussian distributions. Depending on the propagation environment, we should note that both von Mises distribution for the azimuth angle and uniform distribution for the elevation angle perform better. Thus, azimuth distribution function is expressed as :

$$
p\left(\phi_{\mathrm{Tx}}\right)=\frac{\exp \left\{k_{\mathrm{Tx}} \cos \left(\phi_{\mathrm{Tx}}-\bar{\phi}_{\mathrm{Tx}}\right)\right\}}{2 \pi I_{0}\left(k_{\mathrm{Tx}}\right)}
$$

The elevation distribution function is :

$$
p\left(\theta_{\mathrm{Tx}}\right)=\frac{1}{2 \Delta \theta_{\mathrm{Tx}}}
$$

where :

- $\phi_{\mathrm{Tx}} \in[-\pi, \pi]$ 
- $\bar{\phi}_{\mathrm{Tx}}$ is the mean azimuth angle

- $I_{0}(\cdot)$ is the modified Bessel function of zeroth order

- $k_{\mathrm{Tx}}$ is a measure of the azimuth angle dispersion. $1 / k_{\mathrm{Tx}}$ is analogous to the variance in the uniform distribution.

- $\Delta \theta_{\mathrm{Tx}}$ is the standard deviation of the Angle spread

- $\theta_{\mathrm{Tx}} \in\left[\bar{\theta}_{\mathrm{Tx}}-\Delta \theta_{\mathrm{Tx}}, \bar{\theta}_{\mathrm{Tx}}+\Delta \theta_{\mathrm{Tx}}\right]$ where $\bar{\theta}_{\mathrm{Tx}}$ is the mean elevation angle

In the following, we present simulation results of the spatial correlation as a function of the antenna spacing and variable values of the term $f_{\mathrm{Tx}} \xi$. We adopt the MIMO system with two transmit antennas and two receive antennas and we present the plotted curves for the spatial correlation coefficient relative to the transmit antennas $T x_{1}$ and $T x_{2}$. Plotted curves for the spatial correlation are presented in two cases :

1. Single polarized antennas (Fig. 35(a))

2. Dual polarized antennas (Fig. 35(b))

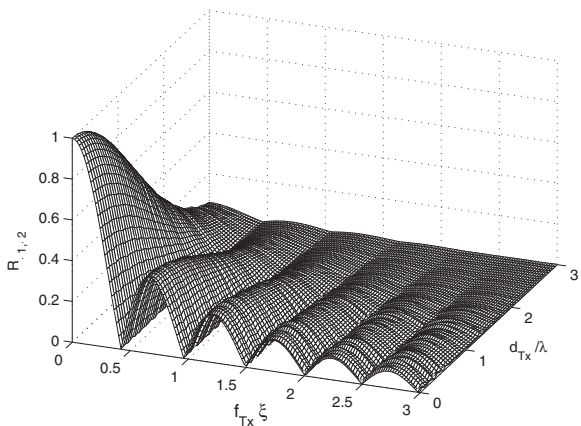

(a)

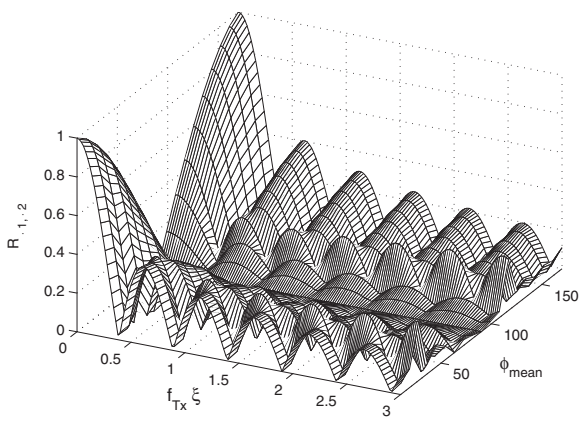

(b)

Fig. 35. (a) Spatial correlation for $\mathrm{z}$ dipole antennas

$\left(k_{\mathrm{Tx}}=50, \bar{\phi}_{\mathrm{Tx}}=90^{\circ}, \bar{\theta}_{\mathrm{Tx}}=90^{\circ}, \Delta \theta_{\mathrm{Tx}}=90^{\circ}\right)$, (b) Spatial correlation for dual polarized antennas ( $\mathrm{z}$ dipole antenna and $\mathrm{x}$ dipole

antenna) $\left(k_{\mathrm{Tx}}=50, \bar{\phi}_{\mathrm{Tx}}=\phi_{\text {mean }}, \bar{\theta}_{\mathrm{Tx}}=90^{\circ}, \Delta \theta_{\mathrm{Tx}}=90^{\circ}\right)$

According to Fig. 35(a) and Fig. 35(b), it is obvious that single polarized antennas system shows less correlation. When dual polarized antennas are deployed, spatial correlation which is expressed as a function of antenna spacing can be reduced. The impact of dual polarization depends on the mean azimuth angle $\bar{\phi}_{\mathrm{Tx}}=\phi_{\text {mean }}$ and spatial correlation is shown to be negligible if $\phi_{\text {mean }}$ approaches $90^{\circ}$. Thus, spatial correlation can be nulled by deploying orthogonal antennas. Finally, we present the impact of dual polarized antennas on MIMO system capacity. The capacity gain brought by the dual polarized system relative to the single polarized system is depicted in Fig. 36. The capacity gain that could be achieved thanks to the use of dual polarized antennas is affected by the mismatch in antenna polarization. Nevertheless, for low XPDs, the dual polarization scheme considerably improves the channel capacity when comparing to the single polarized antennas system. Depolarization phenomena in MIMO system and impact of correlation on MIMO system capacity were rigorously discussed in references (Raoof \& Prayongpun, 2007) and (Raoof \& Prayongpun, 2005). 


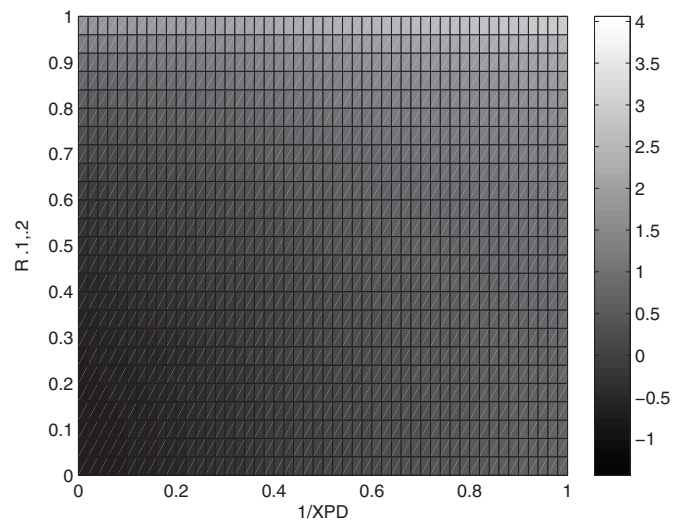

Fig. 36. The capacity gain of $(2 \times 2)$ dual polarized antennas

\section{RSS based localization in WSN with collaborative sensors}

\subsection{Scenario description}

We propose the scenario depicted in Fig. 37 where two clusters of wireless sensor nodes are presented. Cluster 1 consists of a source sensor $S_{T x}$ and a set of $K$ sensors which are randomly distributed in the sensing area.

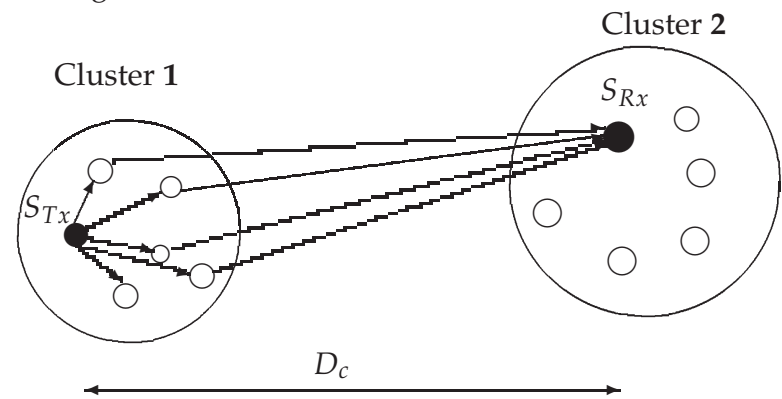

Fig. 37. Scenario description

We assume that sensor nodes are equipped with omnidirectional antennas. The source sensor $S_{T x}$ sends redundant BPSK modulated data signal to the sensor nodes which are located at Cluster 1 . The main problem with the presented scenario is the localization of the sensor $S_{R x}$. We adopt the 2D geometrical model. Sensors are distributed in the (x-y) plan and source node is arbitrary placed at the origin of the system coordinate. The geometric spherical coordinates are defined by the triplet $(r, \theta, \phi)$. Here, $\theta=\frac{\pi}{2}$. The simulated layout for 10 sensors is depicted in Fig.38. We propose a beamforming based localization algorithm involving Received Signal Strength (RSS) measurements. We take into assumption that the radius from the origin to $S_{R x}$ is known. Thus, the detection of the target node relies on determining the steering vector that corresponds to the location of $S_{R x}$. 


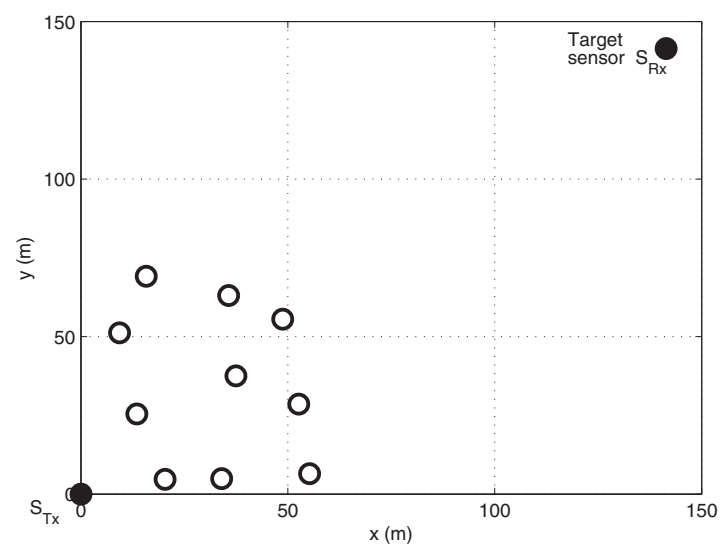

Fig. 38. Network layout $(K=10)$

\subsection{System modeling}

The wireless link between sensor $S_{k}, k=1, \ldots, K$ and the target sensor is modeled by LOS propagation. Each propagation link is characterized by :

1. A Rayleigh distributed attenuation denoted $\lambda_{k}$.

2. A delay $\tau_{k}$ relative to the reference sensor $S_{T x}$ :

$$
\tau_{k}=d_{k} \cdot \frac{\cos \left(\alpha_{k}\right)}{c}
$$

where :

- $d_{k}$ : Distance between sensor $k$ and the source node

- $c$ : Speed of the light

- $\alpha_{k}$ : Direction of arrival relative to the target sensor $S_{R x}$

3. A dephasing $\psi_{k}$ :

$$
\psi_{k}=2 \pi f_{c} \cdot \tau_{k}
$$

$f_{c}$ denotes the frequency carrier.

Each channel gain is therefore expressed as:

$$
\mathbf{h}_{k}=\lambda_{k} e^{j \psi_{k}} \quad ; k=1, \ldots, K
$$

A Hadamard Direct Sequence Code Division Multiple Access (DS-CDMA) code is designed for redundant transmitted BPSK data spreading. Walsh Hadamard codes are perfectly orthogonal and employed to avoid interference among users in the propagation link. These codes are exploited for sensor identification and help to mitigate noise effect. The codes for users could be expressed as the columns (or the rows) of the Walsh-Hamadard matrix $\mathbf{C}$. The simplest Hadamard matrix codes are :

$$
\mathrm{C}_{1}=\mathbf{1}
$$

and

$$
\mathbf{C}_{2}=\left[\begin{array}{cc}
1 & 1 \\
1 & -1
\end{array}\right]
$$


This can be extended to a generic matrix notation using a recursive technique. In fact, if $\mathbf{C}$ is a Hadamard matrix of order $l$ (the spreading code length), then becomes the $2 l$ order Hadamard matrix.

$$
\left[\begin{array}{cc}
C & C \\
C & -C
\end{array}\right]
$$

and

$$
\mathbf{C}_{2^{l}}=\mathbf{C}_{2} \otimes \mathbf{C}_{2^{l-1}}
$$

where $\otimes$ is the Kronecker product and $\mathbf{C}_{2^{l-1}}, 2 \leq l$ is expressed as :

$$
\mathbf{C}_{2^{l}}=\left[\begin{array}{cc}
\mathbf{C}_{2^{l-1}} & \mathbf{C}_{2^{l-1}} \\
\mathbf{C}_{2^{l-1}} & -\mathbf{C}_{2^{l-1}}
\end{array}\right]
$$

Collaborative sensor nodes are presented in Fig. 39.

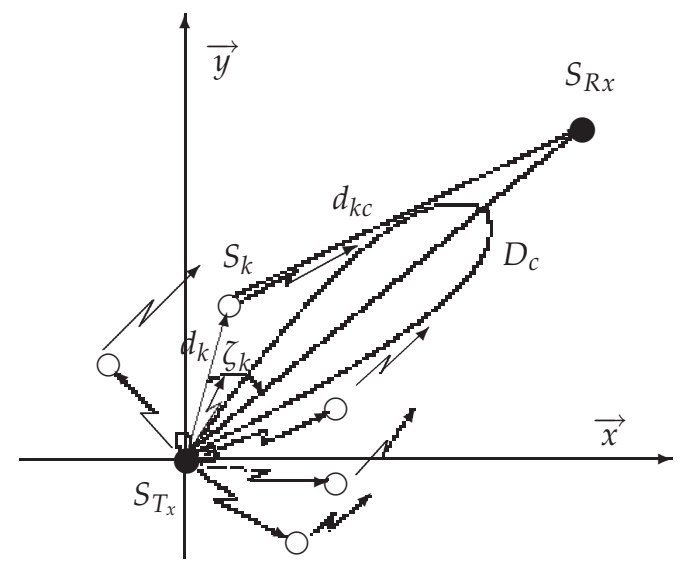

Fig. 39. Collaborative sensor nodes

- $d_{k c}$ : Distance between sensor $k$ and the target node

- $D_{c}$ : Distance between source node and the target sensor

- $\zeta_{k}$ : Direction of transmitted signal vector relative to sensor $k$

Given a sensor $S_{k}$ with cartesian coordinates $\left(x_{k}, y_{k}\right)$, the associate spherical coordinates are :

- $r_{k}=\sqrt{x_{k}^{2}+y_{k}^{2}}$

- $\phi_{k}=\tan ^{-1}\left(\frac{y_{k}}{x_{k}}\right)$

Let $\beta=\frac{2 \pi}{\lambda}$, the steering vector is therefore :

$$
\mathbf{V s}=\left[\exp \left(-j \beta R_{1}\right), \ldots, \exp \left(-j \beta R_{K}\right)\right]
$$

Distance $R_{k}$ is the Euclidean distance between the target sensor with spherical coordinates $\left(D_{c}, \frac{\pi}{2}, \phi_{c}\right)$ and sensor $S_{k}$

$$
R_{k}=\left|D_{c}-d_{k}\right|
$$


The Euclidean distance between points, $\mathrm{A}$ and $\mathrm{B}$ with respective coordinates $A\left(x_{A}, y_{A}\right)$ $B\left(x_{B}, y_{B}\right)$ is :

$$
d_{e u c}=\sqrt{\left(x_{A}-x_{B}\right)^{2}+\left(y_{A}-y_{B}\right)^{2}}
$$

Euclidean distance $R_{k}$ can be approximately expressed as (Ahmed \& Vorobyov, 2009):

$$
R_{k}=\sqrt{D_{c}^{2}+d_{k}^{2}-2 d_{k} D_{c} \cos \left(\phi_{c}-\phi_{k}\right)} \simeq D_{c}-d_{k} \cos \left(\phi_{c}-\phi_{k}\right)=D_{c}-d_{k} \cos \left(\zeta_{k}\right)
$$

\section{RSS based target sensor localization}

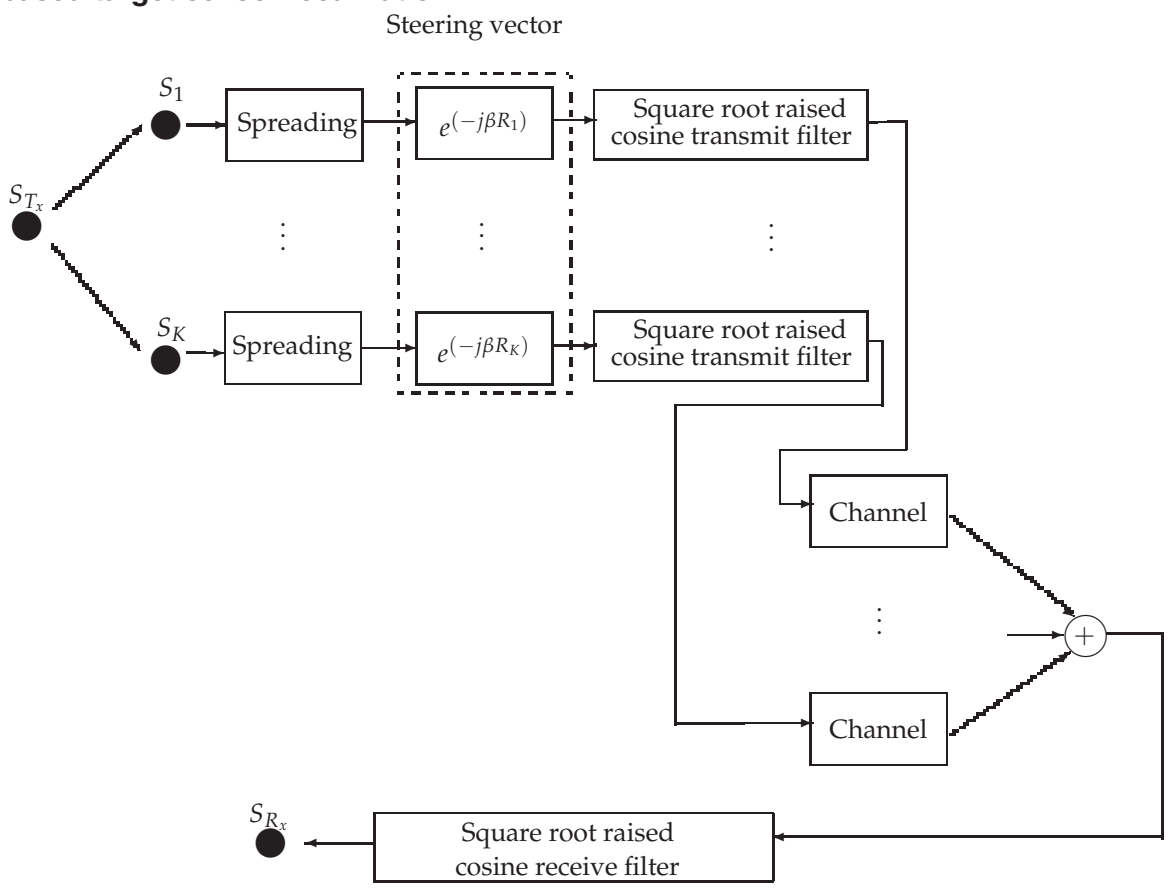

Fig. 40. The transmit location estimation process

The target sensor is said to be detected if the azimuthal coordinate $\phi_{c}$ is accurately estimated. Antennas of sensors $S_{1}, \ldots, S_{K}$ are steered in variable directions relative to a possible location of the target sensor. In this way, the steering vector becomes a function of the angle $\phi_{c}$. The location estimation process starts by finding the location of the sensors within Cluster 1 . The set of $K$ sensor nodes collaboratively form a common message in the direction of the target sensor node $S_{R x}$ (See Fig. 39). The target sensor node feedbacks the CDMA code to sensor nodes within Cluster $\mathbf{1}$. The received data is then despreaded and broadcasted to sensor node $S_{T x}$. The location of the target sensor node is estimated following the RSS measurements performed for all possible locations with respect to a scanning step. The estimated angle of the target sensor corresponds to the maximum measured RSS (measurement of the power present in a received radio signal) value. The received SNR is then evaluated and the accuracy of the proposed algorithm is evaluated in terms of the variation of the statistical parameters as a function of the SNR. The transmit localization process is depicted in Fig. 40. We apply a 
square root cosine matched filtering. The impulse response of the square root cosine filter is shown in Fig. 41.

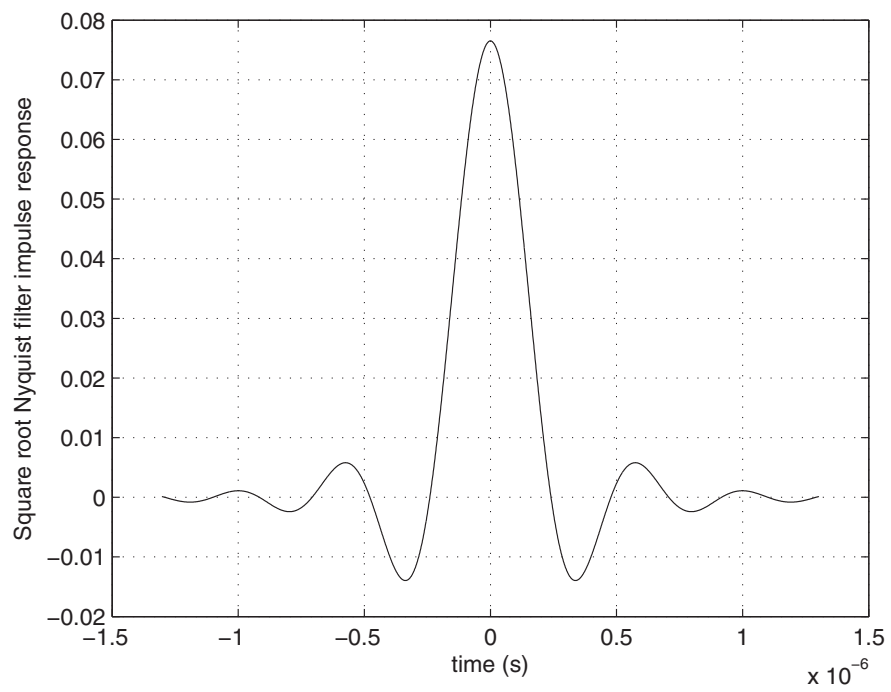

Fig. 41. Square root Nyquist filter

\subsection{Simulation results}

We present in this section, the simulation results in terms of statistical parameters which are obtained following the Monte Carlo method. The main simulation parameters are :

\begin{tabular}{|l|l|}
\hline Simulation parameter & Value \\
\hline \hline Spreading factor & 16 \\
\hline$D_{c}$ & $200 \mathrm{~m}$ \\
\hline Number of angle scanning & 50 \\
\hline Oversampling factor (Nyquist filter) & 8 \\
\hline
\end{tabular}

\section{Mean square error}

The mean square error (MSE) of an estimator is used to quantify the difference between an estimator and the true value of the quantity being estimated.

$$
\operatorname{MSE}\left(\phi_{c}\right)=E\left[\left(\phi_{c}-\bar{\phi}_{c}\right)^{2}\right]
$$

$\overline{\phi_{c}}$ denotes the sample average of $\phi_{c}$.

\section{Statistical error measurement}

Statistical errors are also computed for different number of sensors. This helps to measure the deviation of the estimated location target node from its mean value. Plotted curves for statistical estimator parameters including error estimator and MSE are shown in Fig.43 and Fig.42. These are presented as a function of the received SNR. Simulation results are presented 
for variable numbers of sensors. The obtained results show that the target sensor location is better estimated if more collaborative sensors are used. When 16 sensors are introduced, the error estimation is almost negligible even for low SNR value. We should note that for far target sensor node, we obtain higher error estimation. Fig. 44 shows the plotted curves of the estimator error as a function of SNR for $D=200 \mathrm{~m}$ and $D=400 \mathrm{~m} .8$ collaborative sensors are deployed in the network.

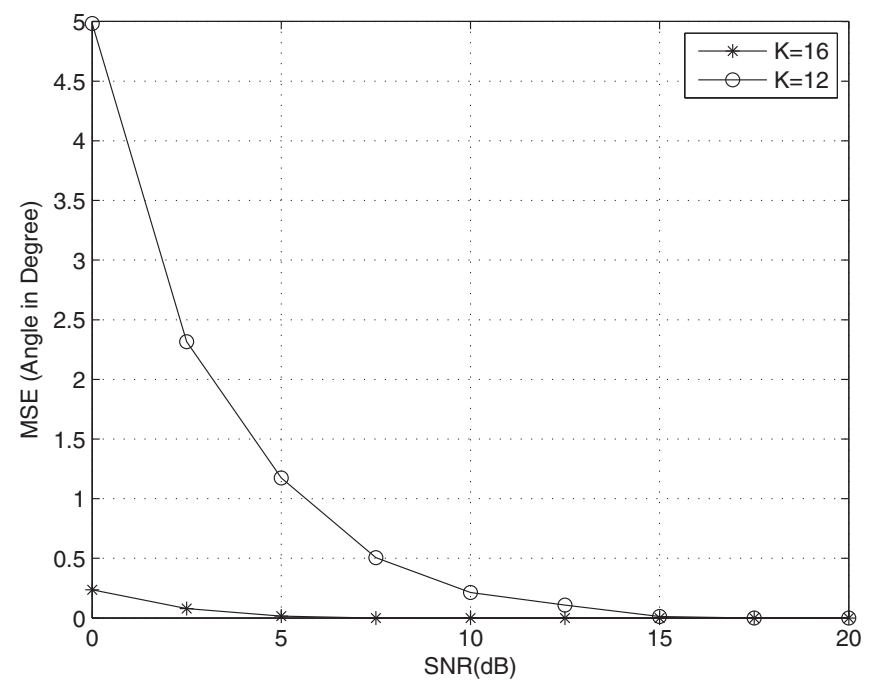

Fig. 42. The mean square error for variable number of sensors

\section{Conclusion}

Throughout this chapter, we showed that MIMO technology brings significant advances in spectral efficiency by employing several antennas at both ends of the communication system. The geometric channel model was rigorously described and correlated MIMO systems were also considered. Correlation occurs in rich scattering environment mainly when antennas are spaced close of each others. In this chapter, we were interested by MIMO processing techniques such as diversity combining, antenna selection and beamforming. Performances of MIMO systems involving combining techniques were evaluated. We showed that selection schemes can reduce the system hardware complexity at the mobile while keeping sufficient performance in MIMO systems. Beamforming processing was also presented. Some beamforming scenarios were studied and simulated in this chapter. Then, polarization diversity for MIMO systems was investigated and depolarization phenomena was studied and quantified by the cross polarization discrimination XPD. When orthogonal antennas are used, the spatial correlation effect can be reduced or eliminated due to low radiation pattern interference. Thus, colocated multi polarized antennas can be deployed. Capacity gain for MIMO systems with dual polarized antennas compared to single polarized antennas was evaluated. Numerical results show that for high XPD values, single polarized antennas perform better. We have finally presented a communication model for WSN involving two 


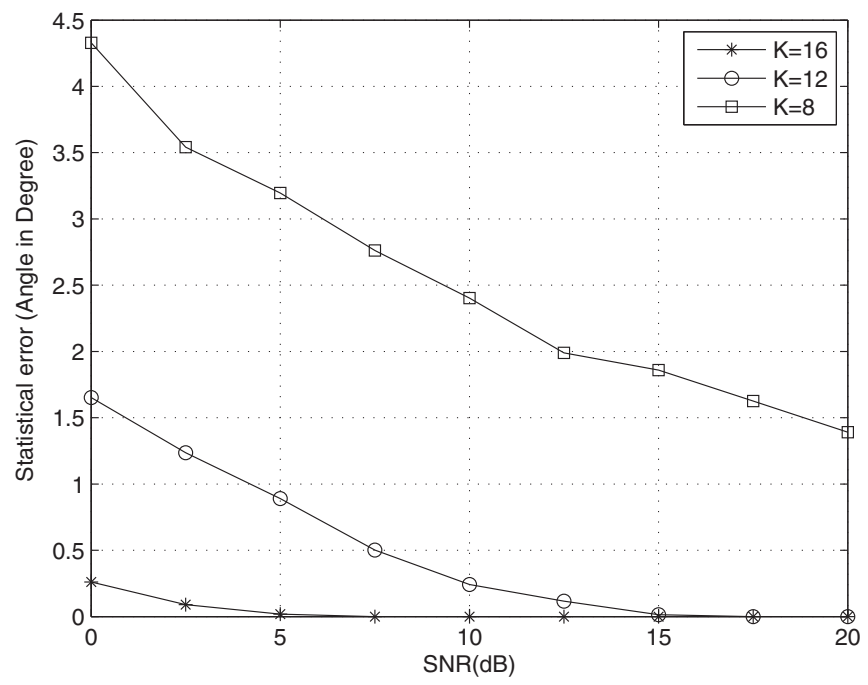

Fig. 43. Statistical error as a function of sensors number $K$

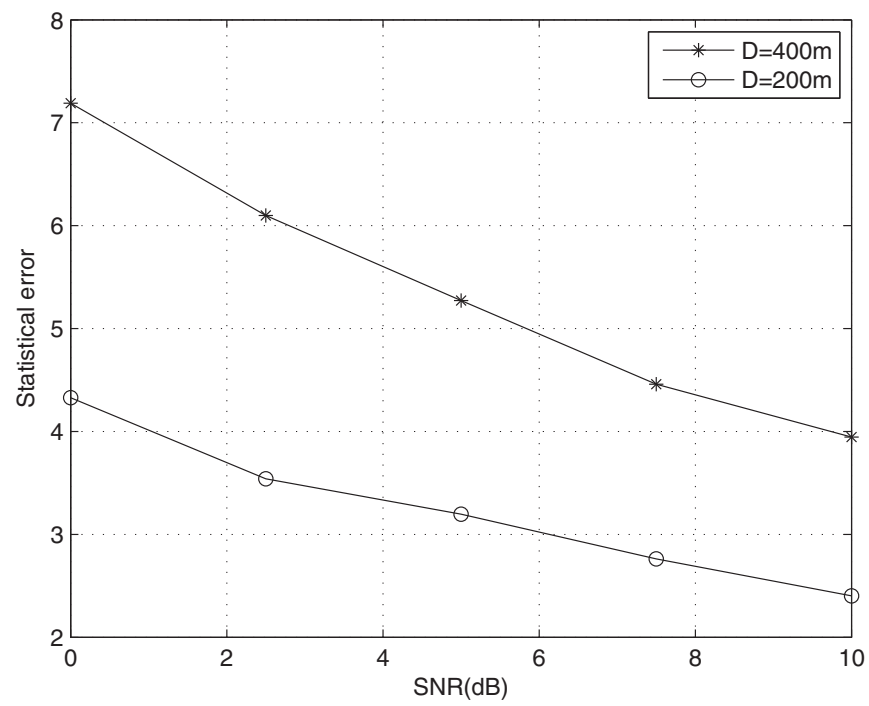

Fig. 44. Impact of distance between the source node and the target node 
clusters of sensors at both the transmit side and the receive side. The set of sensors located at the transmit cluster communicating with sensor nodes located at another cluster perform a distributed MIMO system. RSS based localization exploiting beamforming technique was numerically developed. The mean square error and the estimator variance associated with the target sensor location were evaluated as a function of SNR for various parameters of simulation. The obtained results show that accurate target sensor localization based on beamforming processing and RSS measurement could be achieved if the communication model is properly configured. The performed localization technique could be also carried out under more severe channel environment. Thus, convenient antenna polarization strategy for sensors has to be determined in order to fulfill efficient target sensor localization.

\section{References}

Ahmed, M.F.A. \& Vorobyov, S.A. (2009). Collaborative beamforming for wireless sensor networks with gaussian distributed sensor nodes, IEEE Transactions on Wireless Communications Vol. 8(No. 2): 638-643.

Alamouti, S.M. (1998). A simple transmit diversity technique for wireless communications, IEEE Journal on Selected Areas in Communications Vol. 16(No. 8): 1451-1458.

Ben Zid, M., Raoof, K. \& Bouallègue, A. (2011). Sensor nodes selection in Wireless Sensor Networks over a rich scattering environment, Accepted for presentation at The IEEE 2011 International Conference on Communications, Computing and Control Applications (CCCA'11) and for publication in its proceedings, Tunisia.

Biglieri, E., Calderbank, R., Constantinides, A., Goldsmith, A., Paulraj, A. \& Poor, H. (2007). MIMO Wireless Communications, Cambridge University Press, ISBN: 978-0-521-87328-4.

Burr, A.G. (2003). Capacity bounds and estimates for the finite scatterers MIMO wireless channel, IEEE Journal on Selected Areas in Communications Vol. 21(No. 5): 812-818.

Che-Wei Chang, Kothari, A., Jafri, A., Koutsonikolas, D., Peroulis, D. \& Hu, Y.C. (2008). Radiating sensor selection for distributed beamforming in wireless sensor networks, Proceedings of Military Communications Conference (MILCOM), San Diego, pp. 1-7.

Constantine, A.B. (2005). Antenna Theory: Analysis and Design, John Wiley \& Sons, Ltd, ISBN: 9780471592686, New Jersey.

Dong, Q.T., Prayongpun, N. \& Raoof, K. (2008). Antenna selection for MIMO systems in correlated channels with diversity technique, Proceedings of 4th IEEE International Conference on Wireless Communication, Networking and Mobile Computing (WiCOM), Dalian, pp. 1-4.

Foshini, G.F. (1996). Layered space-time architecture for wireless communication in a fading environment when using multi-element antennas, Bell Labs Technical Journal pp. 41-59.

Freitas, W.C., Cavalcanti, F.R.P. \& Lopes, R.R. (2005). Hybrid transceiver schemes for spatial multiplexing and diversity in MIMO systems, Journal of Communication and Information Systems Vol. 20(No. 3): 63-76.

Ghrayeb, A. (2006). A survey on antenna selection for MIMO communication systems, Information and Communication Technologie (ICTTA) pp. 2104-2109.

Ibnkahla, M. (2009). Adaptive signal processing in wireless communications, CRC Press, ISBN: 978-1-4200-4602-1.

Jafarkhani, H. (2005). Space-Time Coding Theory and Practice, Cambridge University Press, ISBN: 978-0-511-11562-2. 
Marzetta, T.L. \& Hochwald, B.M. (1999). Capacity of a mobile multiple-antenna communication link in Rayleigh flat fading, IEEE Transactions on Information Theory Vol. 45(No. 1): 139-157.

Prayongpun, N. (2009). Modélisation et étude de la capacité du canal pour un système multi-antennes avancé exploitant la diversité de polarisation, Thesis dissertation-UJF-Grenoble I- Kosai RAOOF (Director) .

URL: http://tel.archives-ouvertes.fr/tel-00396666/en/

Proakis, J.G. (1995). Digital Communications, MacGraw-Hill, Inc., ISBN: 0-07-051726-6.

Raleigh, G.G. \& John, J.M. (1998). Spatio-temporal coding for wireless communication, IEEE Transactions on Communications Vol. 46(No. 3): 357-366.

Raoof, K. \& Prayongpun, N. (2005). Channel capacity performance for MIMO polarized diversity systems, Proceedings of 2005 International Conference on Wireless Communications, Networking and Mobile Computing , pp. 1-4.

Raoof, K. \& Prayongpun, N. (2007). Impact of depolarization effects on MIMO polarized wireless configuration, Proceedings of International Conference on Wireless Communications, Networking and Mobile Computing (WiCom), Shanghai, pp. 1-4.

Raoof, K. \& Zhou, H. (2009). Advanced MIMO systems, Scientific Research Publishing, ISBN:978-1-935068-02-0.

Saleh, A. \& Valenzuela, R. (1987). A statistical model for indoor multipath propagation, IEEE Journal on Selected Areas in Communications Vol. 5(No. 2): 128-137.

Sanayei, S. \& Nosratinia, A. (2004). Antenna selection in MIMO systems, IEEE Communications Magazine Vol. 42(No. 10): 68-73.

Shuguang, C., Goldsmith, A.J. \& Bahai, A. (2004). Energy-efficiency of MIMO and cooperative MIMO techniques in sensor networks, IEEE Journal On Selected Areas In Communications Vol. 22(No. 6): 1089-1098.

Tarighat, A., Sadek, M. \& Sayed, A.H. (2005). A multi user beamforming scheme for downlink MIMO channels based on maximizing signal-to-leakage ratios, Proceedings of IEEE International Conference on Acoustics, Speech, and Signal Processing., pp. iii/1129- iii/1132.

Tarokh, V., Jafarkhani, H. \& Calderbank, A.R. (1999). Space-time block codes from orthogonal designs, IEEE Transactions on Information Theory Vol. 45(No.5): 1456-1467.

Tarokh, V., Seshadri, N. \& Calderbank, A.R. (1998). Space-time codes for high data rate wireless communication: Performance criterion and code construction, IEEE Transactions on Information Theory Vol. 44(No. 2): 744-765.

Telatar, I. E. (1995). Capacity of multi-antenna gaussian channels, European Transactions on Telecommunications Vol. 10(No. 6): 585-595.

Tran, T.A. \& Sesay, A.B. (2003). Orthogonal space-time block codes: performance analysis and comparisons, Proceedings of Information, Communications and Signal Processing and the Fourth Pacific Rim Conference on Multimedia, pp. 1497-1501.

Vucetic, B. \& Yuan, J. (2003). Space-Time Coding, John Wiley \& Sons, Ltd, ISBN: 0-470-84757-3.

Wang, C., Hong, X., Ge, X., Cheng, X., Zhang, G. \& Thompson, J. (2010). Cooperative MIMO channel models: A survey, IEEE Communications Magazine Vol. 48(No. 2): 80-87.

Winters, J. (1987). On the capacity of radio communication systems with diversity in a Rayleigh fading environment, IEEE Journal on Selected Areas in Communications Vol. 5(No. 5): 871-878.

Yu, W., Cioffi, J. (2001). On constant power water-filling, Proceedings of IEEE International Conference on Communications (ICC), Helsinki, pp. 1665-1669. 
Yu, C., Ding, Z. \& Chiueh, T. (2004). Design and simulation of a MIMO OFDM baseband transceiver for high throughput wireless LAN, Proceedings of The 2004 IEEE Asia-Pacific Conference on Circuits and Systems, Taiwan, pp. $205-208$.

Zheng, L. \& Tse, D.N.C. (2003). Diversity and multiplexing: a fundamental tradeoff in multiple-antenna channels, IEEE Transactions on Information Theory Vol. 49(No. 5): 1073-1096.

Zhou, H. \& Okamoto, K. (2004). Comparison of code combining and MRC diversity reception in mobile communications, Proceedings of 2004 IEEE Wireless Communications and Networking Conference (WCNC), pp. 943-947. 


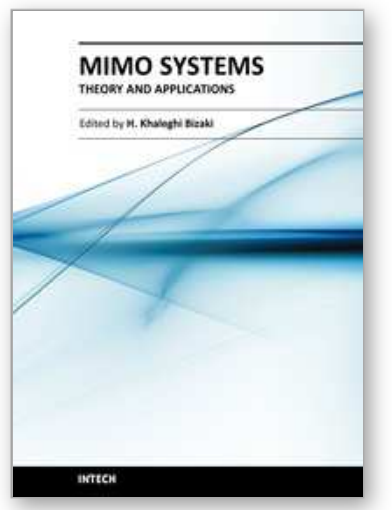

\author{
MIMO Systems, Theory and Applications \\ Edited by Dr. Hossein Khaleghi Bizaki
}

ISBN 978-953-307-245-6

Hard cover, 488 pages

Publisher InTech

Published online 04, April, 2011

Published in print edition April, 2011

In recent years, it was realized that the MIMO communication systems seems to be inevitable in accelerated evolution of high data rates applications due to their potential to dramatically increase the spectral efficiency and simultaneously sending individual information to the corresponding users in wireless systems. This book, intends to provide highlights of the current research topics in the field of MIMO system, to offer a snapshot of the recent advances and major issues faced today by the researchers in the MIMO related areas. The book is written by specialists working in universities and research centers all over the world to cover the fundamental principles and main advanced topics on high data rates wireless communications systems over MIMO channels. Moreover, the book has the advantage of providing a collection of applications that are completely independent and self-contained; thus, the interested reader can choose any chapter and skip to another without losing continuity.

\title{
How to reference
}

In order to correctly reference this scholarly work, feel free to copy and paste the following:

Kosai Raoof, Maha Ben Zid, Nuttapol Prayongpun and Ammar Bouallegue (2011). Advanced MIMO Techniques: Polarization Diversity and Antenna Selection, MIMO Systems, Theory and Applications, Dr. Hossein Khaleghi Bizaki (Ed.), ISBN: 978-953-307-245-6, InTech, Available from:

http://www.intechopen.com/books/mimo-systems-theory-and-applications/advanced-mimo-techniquespolarization-diversity-and-antenna-selection

\section{INTECH}

open science | open minds

\section{InTech Europe}

University Campus STeP Ri

Slavka Krautzeka 83/A

51000 Rijeka, Croatia

Phone: +385 (51) 770447

Fax: +385 (51) 686166

www.intechopen.com

\section{InTech China}

Unit 405, Office Block, Hotel Equatorial Shanghai

No.65, Yan An Road (West), Shanghai, 200040, China

中国上海市延安西路65号上海国际贵都大饭店办公楼405单元

Phone: +86-21-62489820

Fax: $+86-21-62489821$ 
(C) 2011 The Author(s). Licensee IntechOpen. This chapter is distributed under the terms of the Creative Commons Attribution-NonCommercialShareAlike-3.0 License, which permits use, distribution and reproduction for non-commercial purposes, provided the original is properly cited and derivative works building on this content are distributed under the same license. 